\title{
Mean field games and applications
}

\author{
Olivier Guéant, Jean-Michel Lasry, Pierre-Louis Lions
}

\section{Contents}

1 Introduction to mean field games $\quad 2$

1.1 Three routes .................. . . 3

1.1.1 First route: from physics to mean field games . . . . . . . 3

1.1.2 Second route: from game theory to mean field games . . . 5

1.1.3 Third route: from economics to mean field games . . . . . 6

1.2 Fields of application . . . . . . . . . . . . . . . 7

1.2.1 Mean field games versus N-player modeling . . . . . . . . 7

1.2.2 A large family of examples . . . . . . . . . . . . 8

1.3 The mathematical tools of the mean field approach . . . . . . . . 9

2 A first toy model: What time does the meeting start? 9

2.1 An initial simple model . . . . . . . . . . . . . . . 10

2.1.1 Introduction . . . . . . . . . . . . . . . 10

2.1.2 Resolution .................... 11

2.2 Variants . . . . . . . . . . . . . . . . . 13

2.2.1 The framework . . . . . . . . . . . . . . . 14

2.2.2 Existence of an equilibrium for the meeting starting time 15

2.2.3 Comments. . . . . . . . . . . . . . . . 18

2.3 Mean field games equilibrium as the $N$-player Nash limit equilib-

rium ...................... 18

2.3.1 Introduction . . . . . . . . . . . . . . . 18

2.3.2 Solution to $N$-player games . . . . . . . . . . . . . . 19

2.3.3 Approximation in $1 / N \ldots . . . . . . . .222$

3 A mean field game applied to economics: production of an $\begin{array}{ll}\text { exhaustible resource } & 24\end{array}$

3.1 Basis of the model . . . . . . . . . . . . . . . . . . . 24

3.2 The deterministic case . . . . . . . . . . . . . . 25

3.2.1 Characterization of the equilibrium . . . . . . . . 25

3.2 .2 Computation of an equilibrium . . . . . . . . 26

3.2.3 Comments on the deterministic model . . . . . . . . . . . 29

3.3 The stochastic case . . . . . . . . . . . . . . . . . . . . 29

3.3.1 The mean field games PDEs . . . . . . . . . . . . . . 29

3.3.2 Generalization and Externality ........... . 31 
4 Mexican wave $\quad 31$

4.1 Introduction . . . . . . . . . . . . . . . . . . . . . . . 31

4.2 Mexican wave as a solution . . . . . . . . . . . . . . 33

4.3 Mean field games versus descriptive models . . . . . . . . . . 35

5 A model of population distribution $\quad 36$

5.1 Introduction . . . . . . . . . . . . . . . . . . 36

5.2 The framework . . . . . . . . . . . . . . . . 36

5.3 Stationary solutions . . . . . . . . . . . . . . . 37

5.4 Stability results . . . . . . . . . . . . . . . . . 39

5.4.1 Two notions of stability . . . . . . . . . . . . 39

5.4.2 Eductive stability . . . . . . . . . . . . . . . 39

5.4.3 Eductive stability in the localized case . . . . . . . . . 44

5.5 Numerical methods . . . . . . . . . . . . . . . . . . . . . . 44

5.5.1 Stationary equilibrium ............. 45

5.5 .2 Generalizations . . . . . . . . . . . . . 46

6 Asset managers and ranking effect $\quad 47$

6.1 Introduction . . . . . . . . . . . . . . . . . . 47

6.2 The model . . . . . . . . . . . . . . . . . . . 47

6.3 Resolution ..................... . . . 48

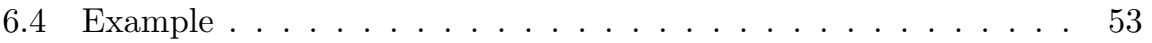

7 Mean field games model of growth and Pareto distribution of salaries $\quad \mathbf{5 4}$

7.1 Introduction to the model mechanisms . . . . . . . . . . . . . . 54

7.2 The optimization problem and the associated PDEs . . . . . . 55

7.3 Solution .................... 56

7.4 Underlying mechanisms ................ 57

7.5 A stochastic generalization .............. 58

8 Mathematical perspectives $\quad 59$ 


\section{About this text ${ }^{1}$ :}

This text is inspired from a "Cours Bachelier" held in January 2009 and taught by Jean-Michel Lasry. This course was based upon the articles of the three authors and upon unpublished materials developed by the authors. Proofs were not presented during the conferences and are now available. So are some issues that were only rapidly tackled during class.

The content of this text is therefore far more important than the actual "Cours Bachelier" conferences, though the guiding principle is the same and consists in a progressive introduction of the concepts, methodologies and mathematical tools of mean field games theory.

Mean field games theory was created in 2006 by Jean-Michel Lasry and PierreLouis Lions and the first results and developments are given in the publications [34, 35, 36]: structures, concepts, definitions of equilibria, forward-backward Hamilton-Jacobi-Bellman/Kolmogorov equation systems, existence theorems in static and dynamic cases, links with Nash equilibria and dynamics in n-player games theory when $n$ tends to infinity, variational principle for decentralization, etc. A number of developments were then implemented by Jean-Michel Lasry and Pierre-Louis Lions, several of them in collaboration with Olivier Guéant: notions of stability of solutions, specific numerical methods, numerical eductive algorithms, and developments in $1 / n$ for a better approximation to n-player games. These developments were presented in three successive courses at the Collège de France [38], in a Bachelier course, in various publications [23, 24] and in Olivier Guéant's PhD thesis [23]. Various applications, notably on the economics of scarce resources, were implemented or are ongoing (in collaboration: Pierre Noël Giraud, Olivier Guéant, Jean-Michel Lasry, Pierre-Louis Lions). Advances in population dynamics were made by Olivier Guéant [23]. Since 2008, several other authors have made further contributions, or are working on new applications and/or properties of MFG models [33, 21].

\section{Introduction to mean field games}

Mean field games theory is a branch of game theory. It is therefore a set of concepts, mathematical tools, theorems, simulation methods and algorithms, which like all game theory is intended to help specialists model situations of agents who take decisions in a context of strategic interactions. These specialists, as in other areas of game theory, will probably be economists, micro- or macroeconomists and, given the specificities of mean field games theory, possibly also sociologists, engineers and even architects or urban planners. In any case, this

\footnotetext{
${ }^{1}$ The research results presented in this text could not have been made without the financial support of the Chair "Finance and Sustainable Development". Hence, the authors express their gratitude to the Chair and all the associated partners.

Also, the "Conseil Français de l'Energie" deserves a special acknowledgment for its specific financial support on the mean field games application to exhaustible resources.
} 
view of the field of application emerges, we feel, from the panorama created by the first "toy models" presented in this text.

We choose the term "toy models" to indicate the particular status of game theory and of many "examples" of it. Consider the famous "prisoner's dilemma". Nobody thinks of taking the story literally, nor that this example was created to be applied to the real-life situations it is supposed to evoke. In fact it is a fable intended to introduce an archetype of strategic interaction: an archetype that can thus be recognized in many negotiation situations in business life and elsewhere. Many of our examples have a similar status. "What time does the meeting start?" or the "Mexican wave equation" should not be taken literally, as a desire to scientifically model these situations in social life. Even if there is clearly an element of truth in our models for these two examples, we believe that the interest for the reader is primarily in the structure that is indicated through these "toy models". The Mexican wave equation, for example, shows how a sophisticated propagation phenomenon in social space can be constructed from non-cooperative individual behaviors in a rational expectation context, once a certain taste for imitation is present in agents' utility function.

Introducing mean field games through these "toy models" is also a way of leading the reader to progressively discover the concepts and the mathematics of mean field games theory.

In this text we present a large amount of results and mathematical proofs. Nevertheless we cover only some parts of the large mathematical corpus built up since 2006. Thus for mathematicians this course can be seen as an introduction, or a reading in parallel with mean field games mathematical papers and with the three different courses held by Pierre-Louis Lions at the Collège de France (06-07, 07-08, 08-09), which present the whole mathematical corpus and which can be downloaded from the Collège de France website [38].

\section{$1.1 \quad$ Three routes}

There are three routes leading naturally to mean field games theory. Each route casts light on a particular aspect of mean field games theory, and the three complement each other.

\subsubsection{First route: from physics to mean field games}

The first route takes its departure from particle physics ${ }^{2}$. In particle physics, mean field theory designates a highly effective methodology for handling a wide

\footnotetext{
${ }^{2}$ Several articles were written using the mean field notion of physicists and applying it to economic dynamics. One may see [15], [16] or [27] as instances of such an approach. Our approach is different from the approach of the "econophysicists" since we are more influenced by control theory and hence more keen on mixing optimization and mean fields. As a consequence, the forward/backward structure of our approach is not present in most of the preceding works.
} 
variety of situations in which there are too many particles to permit the dynamics or equilibrium to be described by modeling all the inter-particle interactions. The enormous number of all these interactions makes a detailed model ineffective: unreadable and unsuitable for both calculation and simulation, the model becomes, in a word, unusable.

Nevertheless, in many situations of this kind, it is possible to construct an excellent approximation to the situation by introducing one or more "mean fields" that serve as mediators for describing inter-particle interactions. In this kind of model, one describes the contribution of each particle to the creation of a mean field, and the effect of the mean field on each particle, by conceiving each particle as infinitesimal, i.e. by carrying out a kind of limit process on the number $n$ of particles $(n \rightarrow \infty)$.

A large proportion of types of inter-particle interactions, though not all, lend themselves to this methodology: the inter-particle interactions must be sufficiently "weak" or "regular" in order for the statistical phenomena to emerge.

Mean field games theory provides an adaptation of this methodology to cases in which the particles are replaced by agents who mutually interact in socioeconomic and/or strategic situations. The main difference, indeed the challenge, is to take account not only of the ability of agents to take decisions, but especially the capacity for strategic interaction, i.e. the capacity of each agent to construct strategies that involve thinking about his peers, who in turn and at the same time elaborate their own individual strategies.

This new sophistication changes the nature of the mean field: it is no longer a statistic on the domain of particle states, but rather a statistic on the domain of agent states and hence on the domain of strategies and information.

This first route is certainly the one that sheds most light on the operating characteristics of mean field games theory: since the methodology of mean fields works very well in particle physics and provides tractable models in a priori complex situations, it offers good prospects for transposition to the world of agents.

But this route is also the most demanding, and would probably be the most discouraging on any initial attempt. Mean field games theory has been able to emerge only because $N$-player game theory has long existed, thanks to the remarkable pioneering work carried out by Von Neumann and Morgenstern ([47]) sixty years ago and to the important developments made since then, notably by Nash $([44,43,45,46])$ and then Aumann $([9])$, and through the many applications that have been developed, particularly in most branches of economics.

What we want to provide is indeed a new branch of game theory for large games that relies on Nash equilibria and on the various concepts introduced in this 
field during the last 50 years. We are not applying, mutatis mutandis, the tools of statistical physics to economic problems. This is an important difference between mean field games and econophysics and we need to insist on that. Econophysicists only apply theories and methods originally rooted in physics to describe an economy and, although they often manage to have good models for the topics under scrutiny, these models are only descriptive. For instance, econophysicists manage to have good descriptive models for wealth distributions using only kinetic models and microscopic interactions (see [14]) but they never explain why people may want to exchange money as in their models (our last model can be seen as an attempt to model wealth distributions in a different way). Mean field games theory proposes to use the tools of physics but to use them inside the classical economic axiomatic, to explain (and not only to describe) phenomenon. Hence we will assign rationality to agents and not regard them as just gas particles, and not even as robots applying some predetermined behavioral strategy: strategic choices are endogenous in our models as they are in game theory.

\subsubsection{Second route: from game theory to mean field games}

This route is the most well-charted from a mathematical standpoint: it involves studying the limit of a large class of $N$-player games when $N$ tends to infinity. Usually, differential games with $N$-players can be summed up by an HJB-Isaacs system of PDEs that turns out to be untractable. Fortunately things are simplified, at least for a wide range of games that are symmetrical as far as players are concerned, as the number of players increases, and for deep reasons. Indeed, interindividual complex strategies can no longer be implemented by the players, for each player is progressively lost in the crowd in the eyes of other players when the number of players increases.

More precisely, the class that proves to be best suited to this passage to the limit is that of games in which players of the same kind can be interchanged without altering the game: a form of anonymity of contexts where nothing is dependent on the individual. This hypothesis is particularly appropriate in the modeling of applications when there are a large number of players. From a mathematical standpoint this hypothesis of invariance through permutation is crucial in moving to the limit.

Moving to the limit causes a situation to emerge in which each player has become infinitesimal amidst the mass of other players, and constructs his strategies from his own state and from the state of the infinite mass of his co-players, who in turn simultaneously construct their strategies in the same way. It is this equilibrium of each player in the mass of co-players that we term the mean field approach.

Continuums of players are not new in the literature and they have been widely used since Robert Aumann and his seminal paper on general equilibrium with 
infinitely many players (see [9]). However, our approach is different in many ways from what has been studied by now (see the literature on large games for instance - $[32,29,30,31])$. An example is the set of specific partial differential equations systems developed and studied in the seminal articles [34, 35, 36] and in [38]. Another instance is the approximation of a $N$-player game by a mean field game and the study on the error term in $\frac{1}{N}$ (see our first toy model). This strategy of approximation allows us to constrain the strategies of the players (since no complex strategies involving specific players can be played) and hence to reduce the finite dimension of the game to a granularity effect that leads to a common noise for the group of players.

\subsubsection{Third route: from economics to mean field games}

In the theory of general economic equilibrium, agents have little concerned about each others: everyone looks only to his own interest and to market prices. The only level at which the existence of others applies is found in the hypothesis of rational expectations. A theory is viewed as credible from the standpoint of rational expectations only if each agent can check whether by putting himself in the place of others he would find the behavior predicted by the theory. This is the only requirement that removes the agent of general equilibrium from his solipsism. In other words, in the theory of general equilibrium, prices mediate all social interactions. Yet we know that in many cases there are other economic effects which give rise to other interactions between agents: externality, public goods, etc. The incorporation of these effects when they are of a statistical nature, which is most often the case, leads to a "mean field"-type definition (in the sense given above) of equilibrium between agents. Similarly, the issues of industrial economics in which agents are involved in complex systems of signaling, entry barriers, positioning in relation to the competition, etc. can become mean field games equilibria when the size of the groups of agents concerned grows.

These interactions between agents are the main interests of economists. They want to understand how prices form through rational behaviors and the consequence of externality effects. Also, economists are interested in the evolution of an economy and hence they have been spending a lot of time on anticipations and the way prices or, more generally, behaviors form in an intertemporal context. This field of economics is clearly untractable for econophysicists since econophysics only considers forward problems without anticipations except perhaps from a heuristical point of view that makes the backward dimension vanish. That's another difference between mean field games theory and the mean fields of econophysicists: mean field games have a forward/backward structure. In most mean field games models, we try not only to describe but also, and most importantly, to explain a phenomenon using the economic toolbox of utility maximization and rational expectations. Hence mean field games theory appears as a toolbox to be used by economists and not as a new competing paradigm in social sciences that avoid considering the major methodological advances made by economists in the last decades. 


\subsection{Fields of application}

\subsubsection{Mean field games versus N-player modeling}

These three routes place the representation of agents in mean field games theory. They are more sophisticated than the agents of general equilibrium in economics, who as we have seen are largely indifferent to their co-agents and are concerned only with prices. Conversely, the agents of mean field games theory are less sophisticated than the players of $N$-player game theory since they base their strategies only on the statistical state of the mass of co-agents.

Nevertheless, this lesser sophistication of the mean field games agent compared to the $N$-player game theory player produces by way of compensation a wide variety of positive effects in two very different respects: in terms of efficiency on the one hand and of widening the field of application on the other.

\section{As far as efficiency is concerned}

A large part of this efficiency and readability comes from the possibility of deploying the power of differential calculus. This advantage is, moreover, one of the differences between mean field games and other prior developments already mentioned in games with an infinite number of players. These works, which follow on from Robert Aumann's outstanding contribution, basically use measure theories, as we do, to represent the continuum of players, but they only use measure theory. From a mathematical standpoint, mean field games theory takes a completely new direction by opening the door to extensive use of differential calculus. Differential calculus has been one of the most powerful and productive tools for some 300 years and major advances have been done in the last decades in many applied fields outside physics: applications of partial differential equations (PDE) to control problems, Itô or Malliavin stochastic calculus, SPDE, and advanced methods of functional analysis. Mean field games theory has moreover enabled a new and autonomous corpus to be developed in this mathematical field, including at the junction of differential calculus and measure theory, in the form of sophisticated developments in the geometry of Wasserstein spaces.

\section{An enlargement of the field of application: two examples}

i) A substantial gain in relation to $N$-player game theory derives from the ease with which questions of player entries and exits can be dealt with. Indeed, through the representation of players by a continuum, the modeling of the renewal of player generations is no longer a problem. Like 
time and player distribution in space, states are continuous variables, and entries and exits are simple flows whose technical treatment presents no special problems. One can thus implement overlapping generation models without pain.

ii) The emergence of a social dimension in mean field games models, since, in these models, statistical data on other players emerge as fundamental constituents of individual strategies. From this point of view, the approximation of $N$-player games by the mean field games limit with, if need be, the use of the corrective term in $1 / N$, allows this approach to introduce a "social" dimension in regard to players, even in limited groups of, say, a few hundred agents.

In view of the positive effects compared with $N$-player games, it seems quite natural to us to consider mean field games "solutions" to problems of $N$-player games. Consider, for example, an $N$-player game where $N$ is fairly small, in the order of a few dozen, and with player entries and exits. It is very likely that in a large number of cases the mean field games limit $(N \rightarrow \infty)$ provides a good first approximation to the $N$-player solution and that the first term of the development in $1 / N$ is sufficient to described with enough precision the effects due to granularity (produced by the fact that $N$ is finite and rather small).

Thus there is a wide field of application for mean field games models. It ranges from general equilibrium with externality to the Mexican wave, and its center of gravity seems to us, from today's standpoint, to be socioeconomic modeling in a rational expectations context.

\subsubsection{A large family of examples}

To illustrate the different aspects of mean field games theory, and to indicate something of its domains of application, we shall in the follow-up to this course present a series of "toy models". In other words, as we mentioned above, we generally present extremely stylized models, which are not to be taken literally and require being reworked by specialists, but which show the possible architecture of applications to various questions:

- Effects of coordination and interactions in the face of externality (meeting time)

- Production of a limited resource (peak oil, Hotelling's rule)

- Mimicry and propagation of behaviors in the social area (Mexican wave, forenames, fashion, etc.)

- Agoraphobia/agoraphilia, search for identity, etc. (quadratic-Gaussian population models) 
- Distortion of financial management in the presence of a signaling problem (managers and ranking)

- Effects of competition on the dynamics of human capital (Pareto-type distribution of salaries: an example of the positive effect of negative externality).

In the course of presenting these "toy models", the mathematical concepts and methods, indeed the whole mean field games toolbox, will become progressively apparent. The considerations mentioned above will thus acquire substance.

\subsection{The mathematical tools of the mean field approach}

The implementation of the mean field games theory as a modeling methodology led to writing new types of systems of equations, then developing the mathematical apparatus required for handling these equations: theorems for the existence of solutions, numerical calculation algorithms, specific definition of stability, variational principles, etc.

We shall return in the conclusion of this course to the mathematical corpus which the reader will be able to discover through these examples.

\section{A first toy model: What time does the meet- ing start?}

We begin with a "toy model" constructed as a series of episodes, or rather as the old TV show "Double your Money", in which the dramatic tension progressively grows. We shall here adopt the serious true/false-type question: "What time does the meeting start?".

We recall what was previously said in the introduction on the role of "toy models", of which the prisoner's dilemma is typical. Nevertheless, we shall proceed as if it involved a scientific investigation of the subject, by tackling it in an increasingly sophisticated step-by-step manner. We therefore begin with a relatively simple framework, then we progressively add various difficulties to give the model greater depth and at the same time reveal the technological answers provided by the mean field games approach. As this "toy model" is presented, we hope the readers will think that it applies to real examples they are particularly familiar with. In the next section, we shall offer a stylized modeling of oil production over a long period of time that will show how our "toy model" can shed light on more serious contexts. 


\subsection{An initial simple model}

\subsubsection{Introduction}

A meeting scheduled for a certain time $t$ very often only starts several minutes after the scheduled time. The actual time $T$ when the meeting starts depends on the dynamics of the arrival of its participants. If a rule sets the start of the meeting at the point when a certain quorum is reached, this rule sets up a form of strategic interaction between agents. We shall construct a first mean field games approach to this situation.

We consider a meeting with a very large number of participants and we agree to consider them as a continuum of agents (the justification will be provided further on). Our agents are rational and understand the situation. More precisely, all the data that we shall provide pertaining to the problem is common knowledge to the meeting participants.

Three times will be important in this model:

- $t$ the scheduled time of the meeting.

- $\tau^{i}$ the time at which agent $i$ would like to arrive in view of the problem. In reality, we suppose that he will arrive at time $\tilde{\tau}^{i}=\tau^{i}+\sigma^{i} \tilde{\epsilon}^{i}$ where $\tilde{\epsilon}^{i}$ is a normal noise with variance 1 , specific to agent $i$ (hypothesis of idiosyncratic noise $^{3}$ ). More precisely, $\tau^{i}$ is a variable controlled by the agent $i$ and $\sigma^{i} \tilde{\epsilon}^{i}$ is an uncertainty the agent is subject to. These uncertainties and their intensity differ in the population of agents since some agents come a long way to participate in the meeting and others are very close. We will note $m_{0}$ the distribution of $\sigma^{i}$ in the population.

- $T$ the actual time the meeting will start (the rule which sets the meeting starting time $T$ according to the arrival of participants is given further on).

To decide on his arrival time, or at least his intended arrival time $\tau^{i}$, each agent will optimize a total cost that, to simplify things (since it is "toy model"), we assume is made of three components:

- A cost (reputation effect) of lateness in relation to the scheduled time $t$ :

$$
c_{1}(t, T, \tilde{\tau})=\alpha[\tilde{\tau}-t]_{+}
$$

- A cost (personal inconvenience) of lateness in relation to the actual starting time of the meeting $T$ :

$$
c_{2}(t, T, \tilde{\tau})=\beta[\tilde{\tau}-T]_{+}
$$

\footnotetext{
${ }^{3}$ This hypothesis of independence will simplify the equations to determine the equilibrium
} 
- A waiting time cost that corresponds to the time lost waiting to reach time $T$ :

$$
c_{3}(t, T, \tilde{\tau})=\gamma[T-\tilde{\tau}]_{+}
$$

Let us note $c(t, T, \tilde{\tau})$ the sum of these three costs which is a convex function of $\tilde{\tau}$.

We can already make the model more complex by assuming different functions for $c_{1}, c_{2}, c_{3}$ according to the agent, but our goal is precisely the opposite: to make it simple in order to explain the methodology.

\subsubsection{Resolution}

The optimization problem that each agent faces is therefore to minimize his expected total cost. Nash-MFG equilibrium, with rational expectations, presupposes that each agent optimizes by assuming $T$ to be known. $T$ is a priori a random variable but because we consider an infinite number of players, the "law of large numbers" will imply that $T$ is deterministic and we consider a deterministic $T$ from now ${ }^{4}$.

For agent $i$ the problem is therefore:

$$
\tau^{i}=\operatorname{argmin} \mathbb{E}\left[c\left(t, T, \tilde{\tau}^{i}\right)\right], \quad \tilde{\tau}^{i}=\tau^{i}+\sigma^{i} \tilde{\epsilon}^{i}
$$

Here $T$ is the mean field, the exhaustive summary for each agent of the behavior of the others.

The exercise is to show the existence of a fixed point $T$, i.e. to show that individual optimization behaviors, supposing $T$ is known, fully generate the realization of this time $T$.

To show that this equilibrium exists, one must first examine more closely agents' individual choices, which is done simply by obtaining a first-order condition.

Proposition 2.1 (FOC). The optimal $\tau^{i}$ of an agent having a $\sigma$ equal to $\sigma^{i}$ is implicitly defined by:

$$
\alpha \mathcal{N}\left(\frac{\tau^{i}-t}{\sigma^{i}}\right)+(\beta+\gamma) \mathcal{N}\left(\frac{\tau^{i}-T}{\sigma^{i}}\right)=\gamma
$$

where $\mathcal{N}$ is the cumulative distribution function associated to a normal distribution.

\section{Proof:}

The expression to minimize is:

$$
\mathbb{E}\left[\alpha\left[\tilde{\tau}^{i}-t\right]_{+}+\beta\left[\tilde{\tau}^{i}-T\right]_{+}+\gamma\left[T-\tilde{\tau}^{i}\right]_{+}\right]
$$

\footnotetext{
${ }^{4}$ Hence rational expectations are simply perfect expectations.
} 


$$
\begin{gathered}
=\mathbb{E}\left[\alpha\left[\tilde{\tau}^{i}-t\right]_{+}+(\beta+\gamma)\left[\tilde{\tau}^{i}-T\right]_{+}-\gamma\left(\tilde{\tau}^{i}-T\right)\right] \\
=\alpha \mathbb{E}\left(\left[\tau^{i}-t+\sigma^{i} \tilde{\epsilon}^{i}\right]_{+}\right)+(\beta+\gamma) \mathbb{E}\left(\left[\tau^{i}-T+\sigma^{i} \tilde{\epsilon}^{i}\right]_{+}\right)-\gamma\left(\tau^{i}-T\right)
\end{gathered}
$$

The first order condition of the problem is therefore given by:

$$
\begin{gathered}
\alpha \mathbb{P}\left(\tau^{i}-t+\sigma^{i} \tilde{\epsilon}^{i}>0\right)+(\beta+\gamma) \mathbb{P}\left(\tau^{i}-T+\sigma^{i} \tilde{\epsilon}^{i}>0\right)=\gamma \\
\alpha \mathcal{N}\left(\frac{\tau^{i}-t}{\sigma^{i}}\right)+(\beta+\gamma) \mathcal{N}\left(\frac{\tau^{i}-T}{\sigma^{i}}\right)=\gamma
\end{gathered}
$$

Since $\mathcal{N}$ is a strictly monotonic cumulative distribution function and since the 3 parameters $\alpha, \beta$ and $\gamma$ are positive, the existence and uniqueness of $\tau^{i}$ can be deduced easily.

From this characterization of $\tau^{i}$ as a function of $\left(t, T, \sigma^{i}\right)$ we can deduce the dynamics of agents' arrival. For this let us consider first of all the distribution $m_{0}$ of the $\sigma^{i}$ within the continuum. Because of the continuum and the law of large numbers, this distribution is transported by the application $\sigma^{i} \mapsto \tilde{\tau}^{i}$.

If we therefore note $F$ the (deterministic !) cumulative distribution function of the agents' real arrival times, it is natural to establish a rule on the real starting time $T$ from the meeting, which depends on the function $F(\cdot)$. An example is that of a quorum: the meeting starts after the scheduled time and only when a proportion $\theta$ of the participants have arrived.

We then have to prove the existence and uniqueness of a fixed point. Starting from a value $T$, we obtain agents' optimal strategies $\left(\tau^{i}(\cdot ; T)\right)_{i}$. These optimal strategies are the targeted times but each person's arrival time is affected by a noise: we obtain the real arrival times $\left(\tilde{\tau}^{i}(\cdot ; T)\right)_{i}$. Then from the law of large numbers and the hypothesis of the independence of agents' uncertainties, these arrival times are distributed according to $F$, which is deterministic, and $T$ is deduced from $F$ by the meeting starting time rule $\left(T^{*}(F)\right)$, in this case the quorum. This is straightforwardly summarized by the following scheme:

$$
T^{* *}: T \mapsto\left(\tau^{i}(\cdot ; T)\right)_{i} \mapsto\left(\tilde{\tau}^{i}(\cdot ; T)\right)_{i} \mapsto F=F(\cdot ; T) \mapsto T^{*}(F)
$$

The result we obtain is as follows:

Proposition 2.2 (Equilibrium $T$ ). If $\alpha>0, \beta>0, \gamma>0$ and if $0 \notin \overline{\operatorname{supp}\left(m_{0}\right)}$ then $T^{* *}$ is a contraction mapping of $[t ;+\infty[$, and there is a unique solution $T$ to our problem.

Proof: First, let's differentiate with respect to $T$ the first order condition that defines $\tau^{i}$.

$$
\frac{d \tau^{i}}{d T}\left[\alpha \mathcal{N}^{\prime}\left(\frac{\tau^{i}-t}{\sigma^{i}}\right)+(\beta+\gamma) \mathcal{N}^{\prime}\left(\frac{\tau^{i}-T}{\sigma^{i}}\right)\right]=(\beta+\gamma) \mathcal{N}^{\prime}\left(\frac{\tau^{i}-T}{\sigma^{i}}\right)
$$


Since 0 is supposed not to be in the support of $m_{0}$, this leads to $\frac{d}{d T} \tau(t, \sigma ; T) \leq$ $k<1$.

Hence, $\forall T, s, h>0$,

$F(s ; T+h)=\mathbb{P}\left(\tau^{i}\left(\sigma^{i} ; T+h\right)+\sigma^{i} \epsilon^{i} \leq s\right) \geq \mathbb{P}\left(\tau^{i}\left(\sigma^{i} ; T\right)+k h+\sigma^{i} \epsilon^{i} \leq s\right)=F(s-k h ; T)$

Consequently,

$$
\begin{gathered}
T^{*}(F(\cdot ; T+h)) \leq T^{*}(F(\cdot-k h ; T)) \leq T^{*}(F(\cdot ; T))+k h \\
\Rightarrow T^{* *}(T+h)-T^{* *}(T) \leq k h
\end{gathered}
$$

and this proves the result through the contraction mapping theorem.

It is interesting to notice that the quorum case is not special in the sense that the preceding proof only requires the $T$ setting rule $\left(T^{*}: F(\cdot) \mapsto T\right)$ to verify the following properties for the above result to be true.

- $\forall F(\cdot), T^{*}(F(\cdot)) \geq t$ : the meeting never starts before $t$

- (Monotony) Let's consider two cumulative distribution functions $F(\cdot)$ and $G(\cdot)$.

If $F(\cdot) \leq G(\cdot)$ then $T^{*}(F(\cdot)) \geq T^{*}(G(\cdot))$

- (Sub-additivity) $\forall s>0, T^{*}(F(\cdot-s))-T^{*}(F(\cdot)) \leq s$

In the more general case where the cost depends on $F$, strategic interaction no longer simply reduces to time $T$. It is very natural that the social cost for each agent depends on the proportion of participants who are already there when he arrives. In this more general case, $F$ is the mean field: each person makes his decision according to $F$. In return, the decisions construct $F$. From a mathematical standpoint, the fixed point concerns $F$.

\section{$2.2 \quad$ Variants}

There are many possible ways of enriching this initial "toy model". For example, one variant involves considering a shared disturbance in addition to the idiosyncratic disturbances. This is an important variant as it is an example where the dynamics of the population is stochastic. Nonetheless, as it would lead us to too long developments we will not consider this variant here.

The variant we shall present is a "geographical" model, i.e. the agents are initially distributed in different places and must come to where the meeting is being held.

The interest of this variant is that it will show how coupled forward/backward PDEs, which are the core of mean field game theory (in continuous time, with a continuous state space), emerge. 


\subsubsection{The framework}

Thus let us suppose that the agents are distributed on the negative half-line according to distribution function $m_{0}(\cdot)$ (with compact support and such that $\left.m_{0}(0)=0\right)$ and that they must go to the meeting held at 0. Suppose that in order to get to 0 , an agent $i$ moves according to the process $d X_{t}^{i}=a_{t}^{i} d t+\sigma d W_{t}^{i}$ where drift $a$ is controlled in return for a quadratic cost $\frac{1}{2} a^{2}$ (here $\sigma$ is the same for everyone). This distribution hypothesis may seem to be a rather artificial representation in this example of transport uncertainties. In practice, we shall see that it is relatively pertinent in other applications.

Each agent is thus faced with an optimization problem, written as:

$$
\min _{a(\cdot)} \mathbb{E}\left[c\left(t, T, \tilde{\tau}^{i}\right)+\frac{1}{2} \int_{0}^{\tilde{\tau}^{i}} a^{2}(t) d t\right]
$$

with $X_{0}^{i}=x_{0}, \quad d X_{t}^{i}=a_{t}^{i} d t+\sigma d W_{t}^{i}$ and the time to reach 0 is given by $\tilde{\tau}^{i}=\min \left\{s / X_{s}^{i}=0\right\}$.

If one looks for a Nash-MFG equilibrium, one will reason at a given $T$ and each agent's problem is one of stochastic control. We thus have the following Hamilton Jacobi Bellman equation ${ }^{5}$ :

$$
0=\partial_{t} u+\min _{a}\left(a \partial_{x} u+\frac{1}{2} a^{2}\right)+\frac{\sigma^{2}}{2} \partial_{x x}^{2} u
$$

This equation can be written:

$$
\partial_{t} u-\frac{1}{2}\left(\partial_{x} u\right)^{2}+\frac{\sigma^{2}}{2} \partial_{x x}^{2} u=0
$$

The condition at the limit is simply $\forall \tau, u(\tau, 0)=c(t, T, \tau)$, where $T$ is deterministic for the same reason as before. This condition corresponds to the total cost on arrival at the meeting (we will assume that $c$ has the same shape as in the preceding setup but we impose $c$ not to be piecewise-linear but twice continuously differentiable).

The Hamilton Jacobi Bellman equation gives a Bellman function $u$ and hence indicates the optimal behavior of agents for a fixed $T$. This equation is the same for all agents since they have the same cost criterion and differ only in their point of departure at the initial moment. Moreover, the solution here is

\footnotetext{
${ }^{5} \mathrm{As}$ it is often the case in this text, we will consider that the solution of the HamiltonJacobi-Bellman equation is a solution of the optimization problem. In general, if we do not provide any verification result for the solutions of the mean field games partial differential equations, it should be noticed that verifying $(u, m)$ indeed provides a solution of the optimization problem is like verifying $u$, solution of the Hamilton-Jacobi-Bellman equation with $m$ fixed, provides a solution of the optimization problem with $m$ fixed. Hence, there should not be specific tools of verification for mean field games.
} 
Markovian as in most stochastic optimization problems and the strategy, i.e. the optimal drift $a(s, x)=-\partial_{x} u(s, x)$ therefore depends only on the place $x$ and the time $s$. Thus, in particular, it does not depend on the agent concerned: the agents, whom unknown factors lead to the same point $x$ at time $s$, adopt the same strategy, which is natural, since they have the same information, same transport costs and same final criterion. This property simplifies the expression of the problem.

The "law of large numbers" then gives us the distribution $m$ of agents through the Kolmogorov equation ${ }^{6}$. This distribution corresponds to the distribution of players who have not yet arrived at 0 and therefore $m$ loses mass (through 0 ), as agents gradually arrive at the meeting. The dynamics of $m$ is:

$$
\text { (Kolmogorov) } \quad \partial_{t} m+\partial_{x}\left(\left(-\partial_{x} u\right) m\right)=\frac{\sigma^{2}}{2} \partial_{x x}^{2} m
$$

$m(0, \cdot)=m_{0}(\cdot)$ is obviously fixed, and we will try to find a solution with the following "smooth fit" condition: $m(\cdot, 0)=0$.

Moreover, as we have chosen to model the problem by the dynamics of Brownian diffusion, the model must be complemented and restricted to a compact domain. In the proof that follows, we suppose that the domain is $\left[0, T_{\max }\right] \times\left[-X_{\max }, 0\right]$ and the boundary conditions are

$$
u\left(T_{\max }, \cdot\right)=c\left(t, T, T_{\max }\right), \quad u\left(\cdot,-X_{\max }\right)=c\left(t, T, T_{\max }\right), \quad m\left(\cdot,-X_{\max }\right)=0
$$

In this context, the flow reaching 0 (when the agents reach the meeting place) is $s \mapsto-\partial_{x} m(s, 0)$. Thus the cumulative distribution function $F$ of arrival times is defined by

$$
F(s)=-\int_{0}^{s} \partial_{x} m(v, 0) d v
$$

Now, $T$ is fixed by the quorum rule (with let's say $\theta=90 \%$ ) but we impose that it must be in the interval $\left[t, T_{\max }\right]$. In other words:

$$
T= \begin{cases}t, & \text { if } F^{-1}(\theta) \leq t \\ T_{\max }, & \text { if } F\left(T_{\max }\right) \leq \theta \\ F^{-1}(\theta), & \text { otherwise }\end{cases}
$$

\subsubsection{Existence of an equilibrium for the meeting starting time}

As in the first simple case, we need to prove that there is a time $T$ coherent with the (rational) expectations of the agents. We are going to use a fixed point

\footnotetext{
${ }^{6}$ Note that this is based not only on the independence hypothesis of noises but also on the simple structure of noises. For example, if volatility depends on state, the associated elliptic operator would replace the Laplace operator. Also, If noises were not independent, the deterministic partial differential equation would have to be replaced by a stochastic one. For all these developments, we refer to [38]
} 
theorem as before. Indeed, one goes from a given $T$ and deduces $u$. The Kolmogorov equation then gives us $m$ and therefore the arrival flow at 0 . Since the time $T$ in our example is given by the arrival of a proportion $\theta$ of all the agents, it clearly is a matter of fixed point.

Before going deeply in the mathematics, let's introduce some hypotheses:

- We suppose that $T \mapsto c(t, T, \tau)$ is a continuous function

- We suppose that $\tau \mapsto c(t, T, \tau)$ is a $C^{2}$ function.

- We suppose that $m_{0}(0)=m_{0}\left(-X_{\max }\right)=0$. Also, we suppose that $\left|m_{0}^{\prime}(0)\right|>0$ and $\left|m_{0}^{\prime}\left(-X_{\max }\right)\right|>0$

Now, we consider the following scheme (the functional spaces involved in the scheme will be proved to be the right ones in what follows):

$T \mapsto c(t, T, \cdot) \in C^{2} \mapsto u \in C^{2} \mapsto \partial_{x} u \in C^{1} \mapsto m \in C^{1} \mapsto-\partial_{x} m(\cdot, 0) \in C^{0}(\mapsto F) \mapsto T$

Since the scheme is from $\left[t, T_{\max }\right]$ to $\left[t, T_{\max }\right]$, to obtain a fixed point result, we just need to prove that the scheme is continuous.

The first part of the scheme $\left(T \mapsto c(t, T, \cdot) \in C^{2}\right)$ is continuous and well defined by hypothesis. For the second part of the scheme $\left(c(t, T, \cdot) \in C^{2} \mapsto u \in C^{2}\right)$, we just state a lemma:

Lemma 2.3. Let's consider the following PDE:

$$
\partial_{t} u-\frac{1}{2}\left(\partial_{x} u\right)^{2}+\frac{\sigma^{2}}{2} \partial_{x x}^{2} u=0
$$

with the boundary conditions

$u(\cdot, 0)=c(t, T, \cdot) \quad u\left(T_{\max }, \cdot\right)=c\left(t, T_{\max }, T_{\max }\right), \quad u\left(\cdot,-X_{\max }\right)=c\left(t, T_{\max }, T_{\max }\right)$

The solution $u$ is in $C^{2}(] 0, T_{\max }[\times]-X_{\max }, 0[)$ and $\exists K, \forall T \in\left[t, T_{\max }\right], \partial_{x} u$ is a K-Lipschitz function.

Moreover the mapping $c(t, T, \cdot) \in C^{2} \mapsto u \in C^{2}$ is continuous.

Now that we get $u$ and then the control $-\partial_{x} u$ we can turn to the Kolmogorov equation. We state a lemma that is an application of Hopf's principle.

Lemma 2.4. Let's consider the following PDE:

$$
\text { (Kolmogorov) } \quad \partial_{t} m+\partial_{x}(a m)=\frac{\sigma^{2}}{2} \partial_{x x}^{2} m
$$

with $a \in C^{1}$ (and hence Lipschitz) and the boundary conditions $m(0, \cdot)=$ $m_{0}(\cdot), \quad m(\cdot, 0)=0, \quad m\left(\cdot,-X_{\max }\right)=0$ where $m_{0}$ is supposed to verify the 
above hypotheses.

Then the solution $m$ is in $C^{1}\left(\left(0, T_{\max }\right) \times\left(-X_{\max }, 0\right)\right)$ and

$$
\exists \epsilon>0, \inf \left|\partial_{x} m(\cdot, 0)\right| \geq \epsilon
$$

Moreover $\epsilon$ only depends on the Lipschitz constant of the function a.

Also the mapping $a \mapsto m \in C^{1}$ is continuous.

From these two lemmas, we can deduce a third one adapted to our problem. Indeed, since $u$ is a $C^{2}$ function, $a=-\partial_{x} u$ is a Lipschitz function and hence we have a lower bound to the flow arriving at the meeting:

Lemma 2.5.

$$
\exists \epsilon>0, \forall T \in\left[t, T_{\max }\right], \inf \left|\partial_{x} m(\cdot, 0)\right| \geq \epsilon
$$

Now, let's consider the mapping $\Psi:-\partial_{x} m(\cdot, 0) \in C^{0} \mapsto T$, defined above using (here) the quorum rule. We are going to prove that $\Psi$ is continuous as soon as $-\partial_{x} m(\cdot, 0)$ has a strictly positive lower bound.

Lemma 2.6. $\Psi$ is a Lipschitz function on $C^{0}\left(\left[0, T_{\max }\right], \mathbb{R}_{+}^{*}\right)$.

\section{Proof:}

Let's consider two functions $\psi_{1}$ and $\psi_{2}$ that stand for two possible flows of arrival and let's define $\epsilon$ a common lower bound to these two functions. Then, let's define $T_{1}=\Psi\left(\psi_{1}\right)$ and $T_{2}=\Psi\left(\psi_{2}\right)$. If $T_{1}$ and $T_{2}$ are both in $] t, T_{\max }$, then, assuming $T_{1} \leq T_{2}$, we can write:

$$
\begin{gathered}
0=\int_{0}^{T_{1}} \psi_{1}-\int_{0}^{T_{2}} \psi_{2}=\int_{0}^{T_{1}}\left(\psi_{1}-\psi_{2}\right)-\int_{T_{1}}^{T_{2}} \psi_{2} \\
\Rightarrow \epsilon\left(T_{2}-T_{1}\right) \leq \int_{T_{1}}^{T_{2}} \psi_{2}=\int_{0}^{T_{1}}\left(\psi_{1}-\psi_{2}\right) \leq T_{\max }\left|\psi_{1}-\psi_{2}\right|_{\infty}
\end{gathered}
$$

Hence, in this case, the function is Lipschitz.

In all other cases, still assuming $T_{1} \leq T_{2}$, we have instead of an equality the following inequality:

$$
\int_{0}^{T_{1}} \psi_{1}-\int_{0}^{T_{2}} \psi_{2} \geq 0
$$

and the result follows from the same reasoning.

Thus, the function is Lipschitz and hence continuous.

By now, we have proved that the scheme is continuous and therefore, using the Brouwer fixed point theorem we have existence of an equilibrium $T$.

Proposition 2.7 (Existence). The scheme that defines the actual $T$ as a function of the anticipated $T$ is continuous and has at least one fixed point. Hence, there is at least one equilibrium $T$. 


\subsubsection{Comments}

This variant is a good example of a mean field game in continuous time and the mechanism will often be the same. First agents anticipate what will be the dynamics of the community and hence anticipate $m$. Here, the relevant information was captured by $T$ that is a function of $m$ so that they had to anticipate $m$ to anticipate $T$. From this anticipation agents use a backward reasoning described by the Hamilton-Jacobi-Bellman equation. Then, from this equation, individual actions can be plugged into the forward equation (the Kolmogorov equation) to know the actual dynamics of the community implied by individual behaviors. Finally, the rational expectation hypothesis implies that there must be coherence between the anticipated $m$ and the actual $m$.

This forward/backward mechanism is the core of mean field games theory in continuous time and we will see it in action later on.

\subsection{Mean field games equilibrium as the $N$-player Nash limit equilibrium}

\subsubsection{Introduction}

Let us return for the sake of simplicity to our first model. It is now time to come back to the continuum hypothesis by considering the game with $N$ players. To simplify the account, and because it involves a "toy model", we look at the same particular case as above (which is rather technical since the criterion is not regular but is very graphic) in which the meeting begins once a proportion $\theta$ (we shall assume $\theta=90 \%$ for the sake of simplicity) of the participants have arrived (but still we force $T$ to be between times $t$ and $T_{\max }$ ). In addition, let us suppose that all the agents have the same $\sigma$. Various questions then naturally arise:

- Does the $N$-player game have Nash equilibria?

- Is there uniqueness of such equilibria?

- Do $N$-player equilibria tend towards the mean field games equilibrium when $N \rightarrow \infty$ ?

- If need be, is the rate of convergence known?

This case is simple, but it allows - since we shall answer the above questions in the affirmative (in the symmetrical case) - to pave the way for an approximation of an $N$-player game by MFG.

This example of approximation of a $N$-player game through a first order expansion " $G_{0}+\frac{1}{N} G_{1}+\ldots$ ", where (formally) $G_{0}$ is the mean field game and $G_{1}$ the first order correction coefficient, leads to a new type of solution of a $N$-player game equilibrium. The solution of " $G_{0}+\frac{1}{N} G_{1}$ " reflects a strategic 
world in which agents do not care about other agents, individually at least, but only about the population dynamics and a world in which $N$, the number of players, is only entered to take into account the "granularity" of the game and the imperfectness of the continuum hypothesis.

\subsubsection{Solution to $N$-player games}

To simplify, let us say that the number of players is $N=10 k(k=1,2,3, \ldots)$ and thus that the meeting begins with the arrival of the $9 k^{t h}$ player. A given player (let us say player 1) will aim for an arrival time $\tau^{*}$ which should verify (symmetrical Nash equation):

$$
\tau^{*}=\operatorname{argmin}_{\tau^{1}} \mathbb{E}\left[C\left(\tau^{1}+\sigma \tilde{\epsilon}^{1}, \tau^{*}+\sigma \tilde{\epsilon}^{2}, \ldots, \tau^{*}+\sigma \tilde{\epsilon}^{N}\right)\right]
$$

This function $C$ does not really depend on all the components of $\left(\tau^{*}+\sigma \tilde{\epsilon}^{2}, \ldots, \tau^{*}+\right.$

$\left.\sigma \tilde{\epsilon}^{N}\right)$ but only on two statistics of order $\tau^{*}+\sigma \tilde{\epsilon}_{(9 k-1)}$ and $\tau^{*}+\sigma \tilde{\epsilon}_{(9 k)}$ where one has noted $\tilde{\epsilon}_{(r)}$ the $r^{t h}$ element, in the order, in $\left\{\tilde{\epsilon}^{2}, \ldots, \tilde{\epsilon}^{N}\right\}$. Indeed it is obvious that the 90-percentile of $\left(\tau^{1}+\sigma \tilde{\epsilon}^{1}, \tau^{*}+\sigma \tilde{\epsilon}^{2}, \ldots, \tau^{*}+\sigma \tilde{\epsilon}^{N}\right)$ is hidden among $\tau^{1}+\sigma \tilde{\epsilon}^{1}, \tau^{*}+\sigma \tilde{\epsilon}_{(9 k-1)}$ and $\tau^{*}+\sigma \tilde{\epsilon}_{(9 k)}$.

Thus the Nash equilibrium is characterized by:

$$
\tau^{*}=\operatorname{argmin}_{\tau^{1}} \mathbb{E}\left[G\left(\tau^{1}+\sigma \tilde{\epsilon}^{1}, \tau^{*}+\sigma \tilde{y}, \tau^{*}+\sigma \tilde{z}\right)\right]
$$

where $(\tilde{y}, \tilde{z})$ are statistics of order corresponding to the $(9 k-1)^{\text {th }}$ and $9 k^{\text {th }}$ ordered elements of $\left\{\tilde{\epsilon}^{2}, \ldots, \tilde{\epsilon}^{N}\right\}$. Hence, the variables $(\tilde{y}, \tilde{z})$ are independent of $\tilde{\epsilon}^{1}$.

Taking up the initial model, the function $G$ is defined by:

$$
\begin{gathered}
\forall a, \forall b, \forall c \geq b, \quad G(a, b, c)=G\left(a, t \vee b \wedge T_{\text {max }}, t \vee c \wedge T_{\text {max }}\right) \\
\forall b \leq c \in\left[t, T_{\text {max }}\right], G(a, b, c)= \begin{cases}-\gamma(a-b) & a \leq t \\
-\gamma(a-b)+\alpha(a-t) & a \in(t, b] \\
\alpha(a-t) & a \in(b, c] \\
\alpha(a-t)+\beta(a-c) & a>c\end{cases}
\end{gathered}
$$

We have the following property:

Lemma 2.8. $\forall b, \forall c \geq b, a \mapsto G(a, b, c)$ is continuous, piecewise linear and convex.

$G$ is not practical for optimization purposes. Let's introduce $H$ the function $\left(\tau^{1}, b, c\right) \mapsto \int_{-\infty}^{\infty} G\left(\tau^{1}+\sigma x, b, c\right) \mathcal{N}^{\prime}(x) d x$ where $\mathcal{N}$ still is the cumulative distribution function of a normal variable with variance 1 . 
Lemma 2.9. $\forall b \leq c, H$ is a strictly convex function of $\tau^{1}$ that decreases and then increases.

Moreover, we can compute some derivatives $\left(t \leq b \leq c \leq T_{\max }\right)$ :

$$
\begin{gathered}
\partial_{1} H\left(\tau^{1}, b, c\right)=\left[-\gamma \mathcal{N}\left(\frac{b-\tau^{1}}{\sigma}\right)+\alpha\left(1-\mathcal{N}\left(\frac{t-\tau^{1}}{\sigma}\right)\right)+\beta\left(1-\mathcal{N}\left(\frac{c-\tau^{1}}{\sigma}\right)\right)\right] \\
\partial_{11}^{2} H\left(\tau^{1}, b, c\right)=\frac{1}{\sigma}\left[\gamma \mathcal{N}^{\prime}\left(\frac{b-\tau^{1}}{\sigma}\right)+\alpha \mathcal{N}^{\prime}\left(\frac{t-\tau^{1}}{\sigma}\right)+\beta \mathcal{N}^{\prime}\left(\frac{c-\tau^{1}}{\sigma}\right)\right] \\
\partial_{12}^{2} H\left(\tau^{1}, b, c\right)=-\frac{1}{\sigma} \gamma \mathcal{N}^{\prime}\left(\frac{b-\tau^{1}}{\sigma}\right) \\
\partial_{13}^{3} H\left(\tau^{1}, b, c\right)=-\frac{1}{\sigma} \alpha \mathcal{N}^{\prime}\left(\frac{c-\tau^{1}}{\sigma}\right)
\end{gathered}
$$

Proof:

$$
\int_{-\infty}^{\infty} G\left(\tau^{1}+\sigma x, b, c\right) \mathcal{N}^{\prime}(x) d x=\frac{1}{\sigma} \int_{-\infty}^{\infty} G(t, b, c) \mathcal{N}^{\prime}\left(\frac{t-\tau^{1}}{\sigma}\right) d t
$$

Hence, we can differentiate with respect to $\tau^{1}$ and we get:

$\partial_{1} H\left(\tau^{1}, b, c\right)=-\frac{1}{\sigma^{2}} \int_{-\infty}^{\infty} G(t, b, c) \mathcal{N}^{\prime \prime}\left(\frac{t-\tau^{1}}{\sigma}\right) d t=-\frac{1}{\sigma} \int_{-\infty}^{\infty} G\left(\tau^{1}+\sigma x, b, c\right) \mathcal{N}^{\prime \prime}(x) d x$

Then, using derivatives in the distribution sense, we get:

$$
\begin{gathered}
\partial_{1} H\left(\tau^{1}, b, c\right)=\int_{-\infty}^{\infty} \partial_{1} G\left(\tau^{1}+\sigma x, b, c\right) \mathcal{N}^{\prime}(x) d x \\
\partial_{1} H\left(\tau^{1}, b, c\right)=\int_{-\infty}^{\infty}\left[-\gamma 1_{\tau^{1}+\sigma x \leq b}+\alpha 1_{\tau^{1}+\sigma x \geq t}+\beta 1_{\tau^{1}+\sigma x \geq c}\right] \mathcal{N}^{\prime}(x) d x \\
\partial_{1} H\left(\tau^{1}, b, c\right)=\left[-\gamma \mathcal{N}\left(\frac{b-\tau^{1}}{\sigma}\right)+\alpha\left(1-\mathcal{N}\left(\frac{t-\tau^{1}}{\sigma}\right)\right)+\beta\left(1-\mathcal{N}\left(\frac{c-\tau^{1}}{\sigma}\right)\right)\right]
\end{gathered}
$$

We can differentiate once again and we get:

$$
\partial_{11}^{2} H\left(\tau^{1}, b, c\right)=\frac{1}{\sigma}\left[\gamma \mathcal{N}^{\prime}\left(\frac{b-\tau^{1}}{\sigma}\right)+\alpha \mathcal{N}^{\prime}\left(\frac{t-\tau^{1}}{\sigma}\right)+\beta \mathcal{N}^{\prime}\left(\frac{c-\tau^{1}}{\sigma}\right)\right]
$$

This is strictly positive so that $H$ is strictly convex as a function of the first variable. Since $H\left(\tau^{1}, b, c\right)=\mathbb{E}\left[G\left(\tau^{1}+\sigma \tilde{\epsilon}^{1}, b, c\right)\right] \geq G\left(\mathbb{E}\left[\tau^{1}+\sigma \tilde{\epsilon}^{1}\right], b, c\right)=G\left(\tau^{1}, b, c\right)$, $H$ must be decreasing and then increasing.

Other derivatives are straightforwardly given by:

$$
\partial_{12}^{2} H\left(\tau^{1}, b, c\right)=-\frac{1}{\sigma} \gamma \mathcal{N}^{\prime}\left(\frac{b-\tau^{1}}{\sigma}\right)
$$




$$
\partial_{13}^{3} H\left(\tau^{1}, b, c\right)=-\frac{1}{\sigma} \alpha \mathcal{N}^{\prime}\left(\frac{c-\tau^{1}}{\sigma}\right)
$$

Let's now recall that we want to find a symmetrical Nash equilibrium and the condition is given by:

$$
\tau^{*}=\operatorname{argmin}_{\tau^{1}} \mathbb{E}\left[G\left(\tau^{1}+\sigma \tilde{\epsilon}^{1}, \tau^{*}+\sigma \tilde{y}, \tau^{*}+\sigma \tilde{z}\right)\right]
$$

Clearly this can be rewritten using the function $H$ and we get:

$$
\tau^{*}=\operatorname{argmin}_{\tau^{1}} \mathbb{E}\left[H\left(\tau^{1}, \tau^{*}+\sigma \tilde{y}, \tau^{*}+\sigma \tilde{z}\right)\right]
$$

Using the first order condition associated to the preceding minimization we see that we need to better understand the function $\partial_{1} H$. The following lemma will be helpful in what follows because it introduces compactness in the problem:

Lemma 2.10.

$$
B=\left\{\tau^{1} \mid \exists b \leq c, \partial_{1} H\left(\tau^{1}, b, c\right)=0\right\}
$$

is a bounded set.

\section{Proof:}

The set we introduced corresponds to the set of points at which $H$ reaches its minimum for all possible couples $(b, c)$ with $b \leq c$. Because $\forall a, \forall b, \forall c \geq$ $b, \quad G(a, b, c)=G\left(a, t \vee b \wedge T_{\max }, t \vee c \wedge T_{\max }\right)$, the same type of properties applies for $H$ and hence our set $B$ is the same as

$$
\left\{\tau^{1} \mid \exists(b, c), t \leq b \leq c \leq T_{\max }, \partial_{1} H\left(\tau^{1}, b, c\right)=0\right\}
$$

Now, $\partial_{1} H\left(\tau^{1}, b, c\right)=0$ implicitly defines a function $\tau^{1}(b, c)$ that is continuous and hence the set $B$ is compact (and then bounded) as the image of a bounded set $\left(\left\{(b, c), t \leq b \leq c \leq T_{\max }\right\}\right)$ by a continuous mapping.

Let's introduce now the best response function of agent 1 . This function $\Gamma$ is defined as:

$$
\Gamma\left(\tau^{*}\right)=\operatorname{argmin}_{\tau^{1}} \mathbb{E}\left[H\left(\tau^{1}, \tau^{*}+\sigma \tilde{y}, \tau^{*}+\sigma \tilde{z}\right)\right]
$$

Another (though implicit) definition of this function is based on the first order condition:

$$
\mathbb{E}\left[\partial_{1} H\left(\Gamma\left(\tau^{*}\right), \tau^{*}+\sigma \tilde{y}, \tau^{*}+\sigma \tilde{z}\right)\right]=0
$$

Lemma 2.11.

$$
\forall \tau^{*}, \inf B \leq \Gamma\left(\tau^{*}\right) \leq \sup B
$$




\section{Proof:}

Since $H$ is decreasing and then increasing as a function of the first variable, we clearly now that $\forall \xi<\inf B$ :

$$
\mathbb{E}\left[\partial_{1} H\left(\xi, \tau^{*}+\sigma \tilde{y}, \tau^{*}+\sigma \tilde{z}\right)\right]<0
$$

Hence $\inf B \leq \Gamma\left(\tau^{*}\right)$. The other inequality is obtained using the same reasoning.

Since a Nash equilibrium simply is a fixed point of $\Gamma$, we can restrict $\Gamma$ to the set $K=[\inf B, \sup B]$.

If we define $\Gamma_{\mid K}: \tau^{*} \in K \mapsto \Gamma\left(\tau^{*}\right)$, we see that any symmetrical Nash equilibrium must be a fixed point of $\Gamma_{\mid K}$.

Now we have our last lemma before the existence and uniqueness theorem that says:

Lemma 2.12. $\Gamma_{\mid K}$ is a contraction mapping from $K$ to $K$.

Proof:

Let's go back to the implicit definition of the function $\Gamma$ given by $(*)$. Using the implicit function theorem we have:

$$
\Gamma^{\prime}\left(\tau^{*}\right)=-\frac{\mathbb{E}\left[\partial_{12}^{2} H\left(\Gamma\left(\tau^{*}\right), \tau^{*}+\sigma \tilde{y}, \tau^{*}+\sigma \tilde{z}\right)+\partial_{13}^{2} H\left(\Gamma\left(\tau^{*}\right), \tau^{*}+\sigma \tilde{y}, \tau^{*}+\sigma \tilde{z}\right)\right]}{\mathbb{E}\left[\partial_{11}^{2} H\left(\Gamma\left(\tau^{*}\right), \tau^{*}+\sigma \tilde{y}, \tau^{*}+\sigma \tilde{z}\right)\right]}
$$

Since $0<-\partial_{12}^{2} H-\partial_{13}^{2} H<\partial_{11}^{2} H$, we have $0 \leq \Gamma^{\prime}\left(\tau^{*}\right)<1$. Now because $K$ is compact, there exists a constant $\varepsilon>0$ so that $\forall \tau^{*} \in K, \Gamma_{\mid K}^{\prime}\left(\tau^{*}\right) \leq 1-\epsilon$.

Now using a classical fixed point result we have:

Proposition 2.13 (Existence and Uniqueness). There exists a unique symmetrical Nash equilibrium for the game with $N$ players.

Remark: We restrict ourselves to cases where $N=10 \mathrm{k}$ and $\theta=90 \%$ for the sake of simplicity but the preceding result is still true for all $N$ and $\theta$.

\subsubsection{Approximation in $1 / N$}

Before beginning the analysis, recall that the equilibrium is a Dirac measure in the mean field game case since all individuals have the same $\sigma$. We note this equilibrium $\tau_{M F G}^{*}$, and the starting time for the meeting will be (except when a limit is reached) $\tau_{M F G}^{*}+\sigma F^{-1}(\theta)$ where $F$ is here the cumulative distribution function of a normal distribution. 
Thus, rather than being defined by:

$$
\mathbb{E}\left[\partial_{1} H\left(\tau_{N}^{*}, \tau_{N}^{*}+\sigma \tilde{y}, \tau_{N}^{*}+\sigma \tilde{z}\right)\right]=0
$$

the mean field games equilibrium is defined by:

$$
\partial_{1} H\left(\tau_{M F G}^{*}, \tau_{M F G}^{*}+\sigma F^{-1}(\theta), \tau_{M F G}^{*}+\sigma F^{-1}(\theta)\right)=0
$$

We see that there is an advantage in introducing $J$ defined by $J(t, y, z)=$ $\partial_{1} H(t, t+\sigma y, t+\sigma z)$ and that we can then carry out the following Taylor expansion:

$$
\begin{aligned}
& 0=\mathbb{E} J\left(\tau_{N}^{*}, \tilde{y}, \tilde{z}\right)= \underbrace{J\left(\tau_{M F G}^{*}, F^{-1}(\theta), F^{-1}(\theta)\right)}_{=0}+\left(\tau_{N}^{*}-\tau_{M F G}^{*}\right) \partial_{1} J\left(\tau_{M F G}^{*}, F^{-1}(\theta), F^{-1}(\theta)\right) \\
&+ \mathbb{E}\left(\tilde{y}-F^{-1}(\theta)\right) \partial_{2} J\left(\tau_{M F G}^{*}, F^{-1}(\theta), F^{-1}(\theta)\right) \\
&+\mathbb{E}\left(\tilde{z}-F^{-1}(\theta)\right) \partial_{3} J\left(\tau_{M F G}^{*}, F^{-1}(\theta), F^{-1}(\theta)\right) \\
&+ \frac{1}{2} \mathbb{E}\left(\tilde{y}-F^{-1}(\theta)\right)^{2} \partial_{22} J\left(\tau_{M F G}^{*}, F^{-1}(\theta), F^{-1}(\theta)\right) \\
&+ \frac{1}{2} \mathbb{E}\left(\tilde{z}-F^{-1}(\theta)\right)^{2} \partial_{33} J\left(\tau_{M F G}^{*}, F^{-1}(\theta), F^{-1}(\theta)\right) \\
&+\mathbb{E}\left(\tilde{z}-F^{-1}(\theta)\right)\left(\tilde{y}-F^{-1}(\theta)\right) \partial_{23} J\left(\tau_{M F G}^{*}, F^{-1}(\theta), F^{-1}(\theta)\right) \\
& \quad+o\left(\tau_{N}^{*}-\tau_{M F G}^{*}\right)+o(1 / N)
\end{aligned}
$$

Detailed study of the properties of order statistics (see [17]), i.e. the variables $\tilde{y}$ and $\tilde{z}$, show that the convergence of $\tau_{N}^{*}$ toward $\tau_{M F G}^{*}$ occurs in $1 / N$.

Indeed, if we write ${ }^{7}$

$$
\begin{gathered}
\xi=\lim _{N \rightarrow \infty} N \mathbb{E}\left(\tilde{y}-F^{-1}(\theta)\right) \in \mathbb{R} \\
\zeta=\lim _{N \rightarrow \infty} N \mathbb{E}\left(\tilde{z}-F^{-1}(\theta)\right) \in \mathbb{R} \\
v=\lim _{N \rightarrow \infty} N \mathbb{E}\left(\tilde{y}-F^{-1}(\theta)\right)^{2} \\
=\lim _{N \rightarrow \infty} N \mathbb{E}\left(\tilde{z}-F^{-1}(\theta)\right)^{2}=\lim _{N \rightarrow \infty} N \mathbb{E}\left(\tilde{z}-F^{-1}(\theta)\right)^{2} \in \mathbb{R}
\end{gathered}
$$

then we see that

$$
\tau_{N}^{*}=\tau_{M F G}^{*}-\frac{1}{N} \frac{1}{\partial_{1} J}\left[\xi \partial_{2} J+\zeta \partial_{3} J+\frac{v^{2}}{2}\left(\partial_{22} J+\partial_{33} J+2 \partial_{23} J\right)\right]+o\left(\frac{1}{N}\right)
$$

The mean field games framework is therefore an approximation of an $N$-player game when $N$ is large and we know the order of magnitude of the error occurring when we consider a mean field games model for solving an $N$-player game.

\footnotetext{
${ }^{7}$ The fact that these constants exist is not obvious and relies on the properties of order statistics
} 


\section{A mean field game applied to economics: pro- duction of an exhaustible resource}

A fairly typical example of mean field game is that of the production of an exhaustible resource by a continuum of producers. We know from Hotelling's work (see [28]) that there is a rent involved in the production of an exhaustible resource, but it is interesting to examine this in greater depth in a competitive situation and to understand the dynamics of exhaustion of a scarce resource. We therefore present a basic model onto which other models can be grafted. For instance, the model can be improved to take account of a Stackelberg-type competition, to consider the existence of big players (OPEC in the instance we have in mind), etc. It is also a basis for studying important problems such as the entry of new competitors, particularly those who are developing alternative energy sources (see [20] for a complete analysis of this question in a mean field game framework identical to the one developed here. This framework allows for instance to consider with powerful analytical tools the negative effect in terms of carbon emissions of a subsidy to alternative energy producers (see [25]) as in $[18])$.

This example will enable us to show the general character of mean field games PDEs when addressing Forward/Backward problems. It also offers a transparent way of dealing with externality.

\subsection{Basis of the model}

We consider a large number of oil producers, which can be viewed either as wells or from a more macro standpoint as oil companies. The only assumption we make is that there is a sufficiently large number of them and that one can apply simple hypotheses such as that of the continuum (mean field games modeling) and perfect competition (price-taker behavior of agents).

Each of these oil producers initially has a reserve that is termed $R_{0}$. We assume that these reserves are distributed among producers according to an (initial) distribution $m(0, \cdot)$. These reserves will of course contribute to production $q$ such that, for any specific agent, we have $d R(t)=-q(t) d t+\nu R(t) d W_{t}$ where the brownian motion is specific to the agent considered.

Production choices will be made in order to optimize a profit criterion (the same for all agents) of the following form:

$$
\max _{(q(t))_{t}} \mathbb{E} \int_{0}^{\infty}(p(t) q(t)-C(q(t))) e^{-r t} d s \quad \text { s.t. } q(t) \geq 0, R(t) \geq 0
$$

where:

- $C$ is the cost function which we will then write as quadratic: $C(q)=$ $\alpha q+\beta \frac{q^{2}}{2}$. 
- the prices $p$ are determined according to the supply/demand equilibrium on the market at each moment, demand being given by a function $D(t, p)$ at instant $t$ (that could be written $D(t, p)=W e^{\rho t} p^{-\sigma}$ where $W e^{\rho t}$ denotes the total wealth affected by a constant growth rate to model economic growth and where $\sigma$ is the elasticity of demand that can be interpreted in a more general model as the elasticity of substitution between oil and any

other good) and supply is naturally given by the total oil production of the agents.

Our model can be dealt with in the deterministic case or in the stochastic case depending on the value of $\nu$.

We are going to start with the deterministic case where $\nu=0$. In that case, a solution can be found without mean field methods. The mean field methods will be necessary in the stochastic case and the economic equilibrium will appear as a very special case of the PDE system, leaving an empty room to add externality effects and for more complex specifications.

\subsection{The deterministic case}

\subsubsection{Characterization of the equilibrium}

Proposition 3.1 (Equilibrium in the deterministic case). The equilibrium is characterized by the following equations where $p, q$ and $\lambda$ are unknown functions and $R_{0}$ the level of initial oil reserve.

$$
\begin{gathered}
D(s, p(s))=\int q\left(s, R_{0}\right) m_{0}\left(R_{0}\right) d R_{0} \\
q\left(s, R_{0}\right)=\frac{1}{\beta}\left[p(s)-\alpha-\lambda\left(R_{0}\right) e^{r s}\right]_{+} \\
\int_{0}^{\infty} q\left(s, R_{0}\right) d s=R_{0}
\end{gathered}
$$

\section{Proof:}

Let's consider the problem of an oil producer with an oil reserve equal to $R_{0}$. The optimal production levels can be found using a Lagrangian:

$$
\mathcal{L}=\int_{0}^{\infty}(p(s) q(s)-C(q(s))) e^{-r s} d s+\lambda\left(R_{0}-\int_{0}^{\infty} q(s) d s\right)
$$

The first order condition is:

$$
p(s)=C^{\prime}(q(s))+\lambda e^{r s}
$$

where $\lambda e^{r s}$ is the Hotelling rent. Noteworthy, if one considered a monopole, the price would not be "marginal cost + rent" but "marginal cost + rent" multiplied by the usual markup. In other words, the actual rent is increasing with 
the market power.

Now, using our specification for the costs, we get, as long as $q(s)$ is positive:

$$
p(s)-\alpha-\beta q(s)=\lambda e^{r s}
$$

Hence, $q(s)$ is given by:

$$
q(s)=\frac{1}{\beta}\left[p(s)-\alpha-\lambda e^{r s}\right]_{+}
$$

In this equation $\lambda$ depends on the initial oil stock (or reserve) and it will be denoted $\lambda\left(R_{0}\right)$. This lagrangian multiplier is given by the intertemporal constraint that equalizes the whole stream of production and the initial oil reserve:

$$
\int_{0}^{\infty} q\left(s, R_{0}\right) d s=\frac{1}{\beta} \int_{0}^{\infty}\left(p(s)-\alpha-\lambda\left(R_{0}\right) e^{r s}\right)_{+} d s=R_{0}
$$

Now, we need to find the prices that were left unknown. This simply is given by the demand/supply equality.

$$
D(s, p(s))=\int q\left(s, R_{0}\right) m_{0}\left(R_{0}\right) d R_{0}
$$

If we compile all these results we get the 3 equations that characterize the equilibrium.

\subsubsection{Computation of an equilibrium}

Since $q$ only depends on $\lambda(\cdot)$ and $p(\cdot)$ we can totally separate the variables $t$ and $R_{0}$. More precisely, if we consider an eductive algorithm (eductive algorithms will be used later to solve coupled PDEs) we can consider two "guesses" $\lambda(\cdot)$ and $p(\cdot)$ to compute $q(\cdot, \cdot)$ and then update $\lambda(\cdot)$ and $p(\cdot)$ using respectively the constraints $\int_{0}^{\infty} q\left(s, R_{0}\right) d s=R_{0}$ and $D(s, p(s))=\int q\left(s, R_{0}\right) m_{0}\left(R_{0}\right) d R_{0}$.

More precisely, we consider a dynamical system indexed by the variable $\theta$ like the following ${ }^{8}$

$$
\begin{gathered}
\partial_{\theta} p(t, \theta)=D(t, p(t, \theta))-\int q\left(t, R_{0}\right) m_{0}\left(R_{0}\right) d R_{0} \\
\partial_{\theta} \lambda\left(R_{0}, \theta\right)=\int_{0}^{\infty} q\left(t, R_{0}\right) d t-R_{0}
\end{gathered}
$$

where

$$
q\left(t, R_{0}\right)=\frac{1}{\beta}\left[p(t, \theta)-\alpha-\lambda\left(R_{0}, \theta\right) e^{r t}\right]_{+}
$$

Once a dynamical system is chosen, the solution for $R_{0} \mapsto \lambda\left(R_{0}\right)$ and $t \mapsto p(t)$ and hence the productions of all oil producers is obtained by:

${ }^{8}$ The system can be multiplied by the inverse of its Jacobian matrix. Different multiplying factors can also be added to the two equations. 


$$
\begin{aligned}
\lim _{\theta \rightarrow+\infty} p(t, \theta) & =p(t) \\
\lim _{\theta \rightarrow+\infty} \lambda\left(R_{0}, \theta\right) & =\lambda\left(R_{0}\right)
\end{aligned}
$$

As an example we can illustrate the evolution of total oil production in this model where we consider a CES demand function, namely $D(t, p)=W e^{\rho t} p^{-\sigma}$.

We took the following values for the parameters: the interest rate considered by oil producers is $r=5 \%$, the average growth rate of the world economy is $\rho=2 \%$, the initial marginal cost of producing an oil barrel is $\alpha=10, \beta=100$ to model the importance of capacity constraints, $\sigma=1.2$ because oil is not a highly elastic good and $W=40$ to obtain meaningful values in the model. The problem is considered over 150 years and the initial distribution of reserves has the following form:

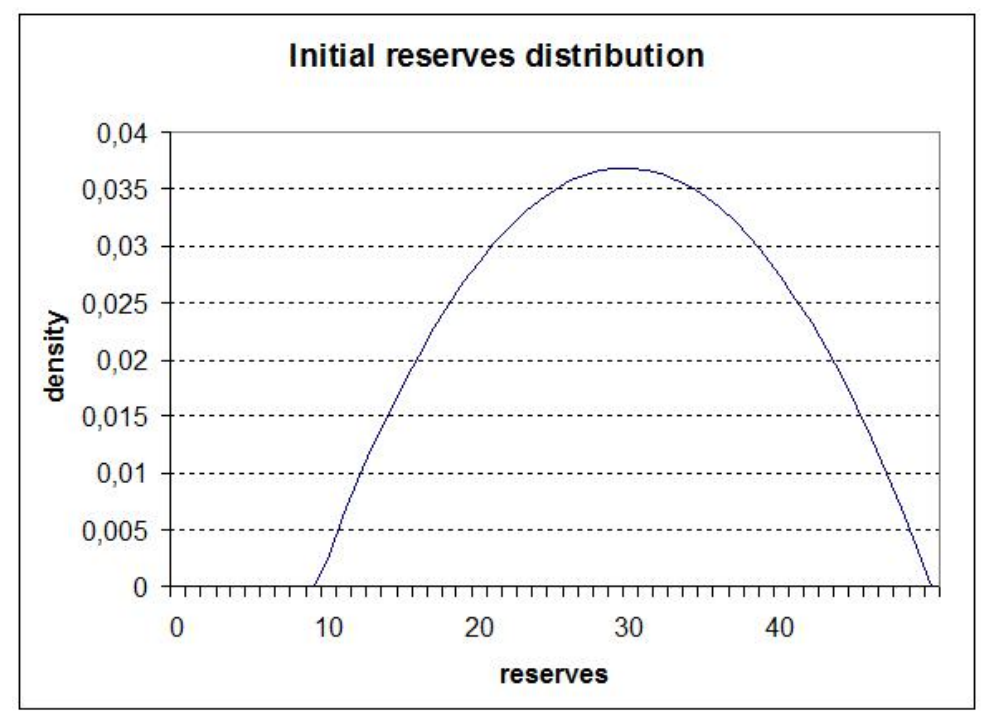

Figure 1: $m_{0}$

If we consider the global production of oil producers, its evolution is given by the first graph below where the horizontal axis represents the years and the vertical one the global production at each date. The associated evolution of oil prices is also represented where we only plot the first 50 years to avoid ending up with very large values after too many decades and hence a graph that is unreadable. 


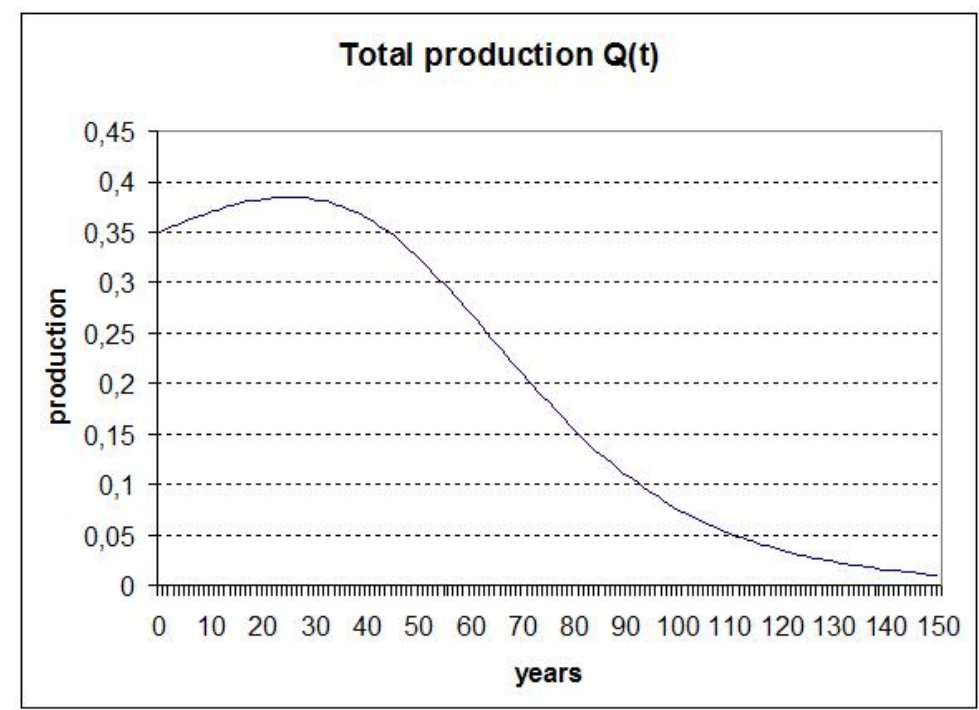

Figure 2: Evolution of the total oil production

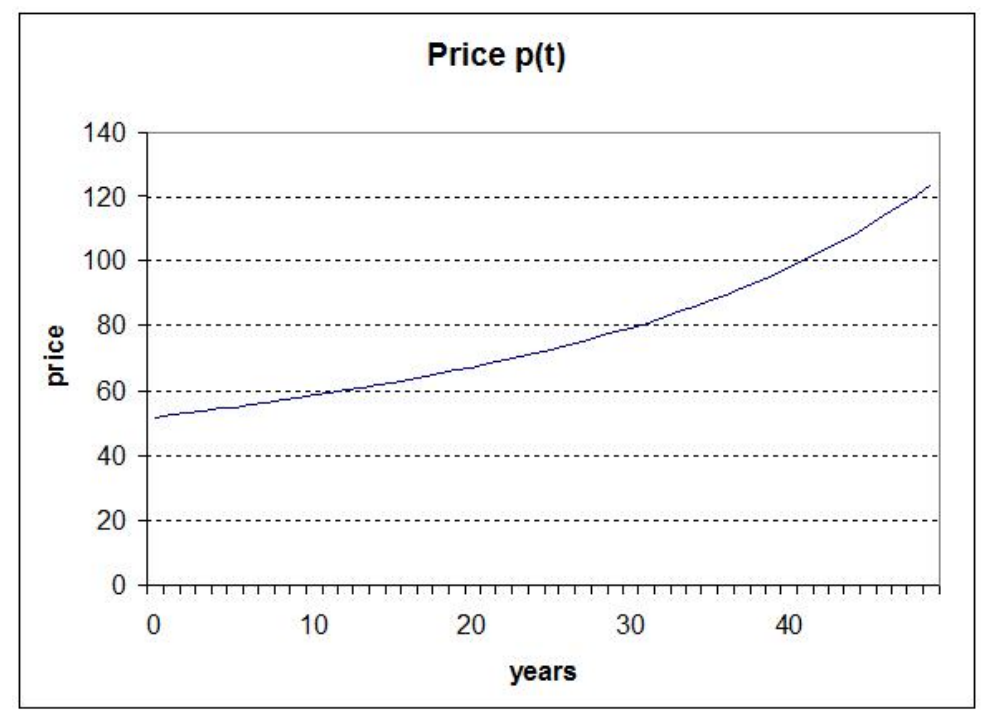

Figure 3: Evolution of prices over 50 years 


\subsubsection{Comments on the deterministic model}

This deterministic model will appear as classical to most readers. Though, some comments deserve to be made. First of all, we saw that prices were not equal to marginal cost since the optimal production (when positive) is given by:

$$
p(t)=C^{\prime}\left(q\left(t, R_{0}\right)\right)+\lambda\left(R_{0}\right) e^{r t}
$$

Hence, the Hotelling rent $\left(\lambda\left(R_{0}\right) e^{r t}\right)$ increases with time and differs among producers. Since $\lambda$ measures the strength of the constraint associated to the exhaustible nature of oil, it is a decreasing function of $R_{0}$. As a consequence, the rent is higher when it comes to consider a smaller producer.

Another remarkable phenomenon is the shape of the curve. Oil production first increases and then decreases. It's a form of the so-called Hubbert peak even though we do not have the symmetry result associated to the usual Hubbert peak $^{9}$.

Economic growth pushes oil producers to produce more (and especially producers with a large oil reserve) but the intrinsic exhaustibility of oil induces a decrease in the production after a certain period of time.

\subsection{The stochastic case}

The above model was a mean field game as any general equilibrium economic model. In the simple deterministic case developed above, the mean field games tools didn't need to be used and classical tools were sufficient, except perhaps when it came to find a numerical solution. However, when it comes to noise or externality in the model, the mean field games partial differential equations will be necessary. In contrast with the PDEs developed for the first toy model, the PDEs will now be completely coupled and not only coupled through boundary conditions.

\subsubsection{The mean field games PDEs}

To start writing the equations, let's introduce $u(t, R)$ the Bellman function of the problem, namely:

$$
\begin{gathered}
u(t, R)=\max _{(q(s))_{s \geq t}, q \geq 0} \mathbb{E} \int_{t}^{\infty}(p(s) q(s)-C(q(s))) e^{-r(s-t)} d s \\
\text { s.t. } d R(s)=-q(s) d s+\nu R(s) d W_{s}, R(t)=R
\end{gathered}
$$

The Hamilton Jacobi Bellman equation associated to this optimal control problem is:

$$
\partial_{t} u(t, R)+\frac{\nu^{2}}{2} R^{2} \partial_{R R}^{2} u(t, R)-r u(t, R)+\max _{q \geq 0}\left(p(t) q-C(q)-q \partial_{R} u(t, R)\right)=0
$$

\footnotetext{
${ }^{9}$ Our model not being suited for it since we do not focus on the discovery and exploitation of new wells.
} 
Now, let's denote $m(t, R)$ the distribution of oil reserves at time $t$. This distribution is transported by the optimal production decisions of the agents $q^{*}(t, R)$ where, now, $R$ is the reserve at time $t$ and not the initial reserve as in the deterministic case.

The transport equation is:

$$
\text { (Kolmogorov) } \quad \partial_{t} m(t, R)+\partial_{R}\left(-q^{*}(t, R) m(t, R)\right)=\frac{\nu^{2}}{2} \partial_{R R}^{2}\left[R^{2} m(t, R)\right]
$$

with $m(0, \cdot)$ given.

Now, let's discuss the interdependence between $u$ and $m$.

$m$ is linked to $u$ quite naturally since $m$ is transported by the optimal decisions of the agents determined by the optimal control in the HJB equation. This optimal control is given by ${ }^{10}$ :

$$
q^{*}(t, R)=\left[\frac{p(t)-\alpha-\partial_{R} u(t, R)}{\beta}\right]_{+}
$$

Now, $u$ depends on $m$ through the price $p(t)$ and this price can be seen as a function of $m$. Indeed, because $p(t)$ is fixed so that supply and demand are equal, $p(t)$ is given by:

$$
p(t)=D(t, \cdot)^{-1}\left(-\frac{d}{d t} \int R m(t, R) d R\right)
$$

If we want to conclude on this part and rewrite the equations to focus on the interdependence, we may write the following expressions:

$$
\begin{gathered}
\partial_{t} u(t, R)+\frac{\nu^{2}}{2} R^{2} \partial_{R R}^{2} u(t, R)-r u(t, R) \\
+\frac{1}{2 \beta}\left[\left(D(t, \cdot)^{-1}\left(-\frac{d}{d t} \int R m(t, R) d R\right)-\alpha-\partial_{R} u(t, R)\right)_{+}\right]^{2}=0 \\
\partial_{t} m(t, R)+\partial_{R}\left(-\left[\frac{D(t, \cdot)^{-1}\left(-\frac{d}{d t} \int R m(t, R) d R\right)-\alpha-\partial_{R} u(t, R)}{\beta}\right]_{+} m(t, R)\right) \\
=\frac{\nu^{2}}{2} \partial_{R R}^{2}\left(R^{2} m(t, R)\right)
\end{gathered}
$$

These equations are the coupled equations associated to our optimization problem but it is still an interesting problem to establish a verification theorem that would prove a solution of the Hamilton-Jacobi-Bellman equation to be a solution of the optimization problem.

\footnotetext{
${ }^{10}$ Once again we suppose that the solution of the (HJB) equation we consider is a solution of the underlying optimization problem. Verification results still need to be proved.
} 


\subsubsection{Generalization and Externality}

The equations, as stated above to focus on the interdependence, are less practical and intuitive than the preceding forms of the equation. Though, they express something really important we want to insist upon: general equilibrium in its classical form can appear as a very special case of a mean field game. A natural consequence is that we can add other effects in a very simple manner while adding meaningful terms to the PDEs.

For example, it's widely thought amongst oil specialists that oil producers not only want to maximize profit but also want to avoid being in the last producers to produce because they do not know what is going to happen to oil companies at the end of the oil era.

This kind of effect would have been very hard to introduce with the first (usual) approach we presented. With the mean field games approach, the addition of such an effect is just another dependence on $m$ in the HJB equation that

defines $u$. One possibility is for example to introduce a ranking effect in the Hamilton Jacobi Bellman equation. The Hamilton Jacobi Belmman equation may become:

$$
\begin{gathered}
\partial_{t} u(t, R)+\frac{\nu^{2}}{2} R^{2} \partial_{R R}^{2} u(t, R)-r u(t, R)+H\left(\int_{0}^{R} m(t, \phi) d \phi\right) \\
+\frac{1}{2 \beta}\left[\left(D(t, \cdot)^{-1}\left(-\frac{d}{d t} \int R m(t, R) d R\right)-\alpha-\partial_{R} u(t, R)\right)_{+}\right]^{2}=0
\end{gathered}
$$

where $H$ is a decreasing function. In addition to the intertemporal profit optimization, the producer wants to have less oil reserve than its competitors.

This generalization is one amongst many. We just aim at convincing the reader about the variety of effects and particularly externality effects the mean field games approach allows to handle quite easily. To see how this mean field game can be adapted to the study of competition between oil producers and potential entrants that produce alternative energy, see [25] and [20].

\section{Mexican wave}

\subsection{Introduction}

Before moving on to more complex models, let us look at a "toy model" which is prompted by mean field games and models the mimicry responsible for the Mexican wave phenomenon in stadiums.

Mexican wave is called this way because it seems that it appeared for the first time in a stadium in Mexico. The goal of our model here is to understand 
how a Mexican wave can be one of the solution of a mean field game involving a (infinite) set of supporters and a taste for mimicry. Let's start with the description of the stadium. To simplify the study, we regard our stadium as a circle of length $L$ (hence the stadium is a one-dimension object, though it wouldn't be difficult to generalize). Mathematically, the stadium will then be the interval $[0, L)$ regarded as a torus.

In the stadium, there is a continuum of individuals; each one being referenced by a coordinate $x \in[0, L)$. Each agent is free to behave and can be either seated $(z=0)$ or standing $(z=1)$ or in an intermediate position $z \in(0,1)$. Some positions are less comfortable than others and we model this phenomenon using a utility function $u$.

Typically $u$ will be of the following form to express that being standing or being seated is more comfortable than being in an intermediate position:

$$
u(z)=-K z^{\alpha}(1-z)^{\beta}
$$

Now, let's describe the optimization function for any agent:

- An agent pays a price $h(a) d t$ to change his position from $z$ to $z+a d t$. $h(a)$ will simply be a quadratic cost function: $\frac{a^{2}}{2}$.

- An agent wants to behave as his neighbors. Mathematically an agent in $x$ maximizes

$$
-\frac{1}{\epsilon^{2}} \int(z(t, x)-z(t, x-y))^{2} \frac{1}{\epsilon} g\left(\frac{y}{\epsilon}\right) d y
$$

where $g$ is a gaussian kernel.

- An agent maximizes his comfort described by $u$.

The optimization criterion for an agent localized at $x$ is then

$\sup _{z(\cdot, x)} \liminf _{T \rightarrow+\infty} \frac{1}{T} \int_{0}^{T}\left\{\left[-\frac{1}{\epsilon^{2}} \int(z(t, x)-z(t, x-y))^{2} \frac{1}{\epsilon} g\left(\frac{y}{\epsilon}\right) d y\right]+u(z(t, x))-\frac{\dot{z}(t, x)^{2}}{2}\right\} d t$

This ergodic control problem can be formally transformed in a differential way and we get:

$$
-\frac{2}{\epsilon^{2}} \int(z(t, x)-z(t, x-y)) \frac{1}{\epsilon} g\left(\frac{y}{\epsilon}\right) d y+u^{\prime}(z(t, x))=-\partial_{t t}^{2} z(t, x)
$$

If we let $\epsilon$ tends to 0 , we get in the distribution sense that our problem is to solve the equation ${ }^{11}$ :

$$
\partial_{t t}^{2} z(t, x)+\partial_{x x}^{2} z(t, x)=-u^{\prime}(z(t, x))
$$

\footnotetext{
${ }^{11}$ This equation doesn't seem to be of the mean field type but we can write the associated mean field equations.

Let's consider that agents are indexed by $x$. For each $x$, the Bellman function associated to
} 
Before going on and solve the problem, we must notice that $z=0$ and $z=1$ should be solutions of the problem. Consequently, we must have $u^{\prime}(0)=u^{\prime}(1)=$ 0 and hence $\alpha$ and $\beta$ have to be strictly greater than 1 .

\subsection{Mexican wave as a solution}

A Mexican wave is, by definition, a wave. Hence we are going to look for a solution of the form $z(t, x)=\phi(x-v t)$ where $v$ is the speed of the wave. But what we call Mexican wave is usually a specific form of wave and we want to call Mexican wave a function $\phi$ with a compact support on $(0, L)$ that is first increasing from 0 to 1 and then decreasing form 1 to 0 .

If we look for such a function $\phi$, we can easily see that it must solve:

$$
\left(1+v^{2}\right) \phi^{\prime \prime}=-u^{\prime}(\phi)
$$

Proposition 4.1 (Existence of Mexican waves for $\alpha, \beta \in(1 ; 2)$ ). Suppose that $\alpha, \beta \in(1 ; 2)$. Then, for any $v$ verifying

$$
\frac{\Gamma\left(1-\frac{\alpha}{2}\right) \Gamma\left(1-\frac{\beta}{2}\right)}{\Gamma\left(2-\frac{\alpha+\beta}{2}\right)}<\sqrt{\frac{K}{2\left(1+v^{2}\right)}} L
$$

there exists a Mexican wave $\phi$ solution of $\left(1+v^{2}\right) \phi^{\prime \prime}=-u^{\prime}(\phi)$.

\section{Proof:}

We use an "energy method" to solve the equation $\left(1+v^{2}\right) \phi^{\prime \prime}=-u^{\prime}(\phi)$. First, let's multiply the equation by $\phi^{\prime}$ and integrate. We get:

$$
\frac{1+v^{2}}{2} \phi^{\prime 2}=-u(\phi)+C
$$

Since $\phi=0$ must be a solution, the constant has to be zero.

the problem of an agent in $x$ can be written as $J(x ; \cdot)$ solving the Hamilton-Jacobi equation:

$$
0=\partial_{t} J(x ; t, z)+\frac{1}{2}\left(\partial_{z} J(x ; t, z)\right)^{2}+u(z)-\frac{1}{\epsilon^{2}} \int(z-\tilde{z})^{2} m(\tilde{x} ; t, \tilde{z}) \frac{1}{\epsilon} g\left(\frac{x-\tilde{x}}{\epsilon}\right) d \tilde{z} d \tilde{x}
$$

where $m(x ; t, \cdot)$ is the probability distribution function of the position $z$ of an agent situated in $x$.

$m(x ; \cdot, \cdot)$ solves a Kolmogorov equation that is:

$$
\partial_{t} m(x ; t, z)+\operatorname{div}\left(\partial_{z} J(x ; t, z) m(x ; t, z)\right)=0
$$

with $m(x ; 0, z)=\delta_{z(0, x)}(z)$ Hence, the problem can be written as a set of Hamilton-Jacobi equations indexed by $x$ with the associated Kolmogorov equations. Because the problem is purely deterministic, we can directly follow the position of each individual and consider an equation in $z(t, x)$ instead of this complex system. 
Consequently, we are left with an ODE:

$$
\phi^{\prime}= \pm \sqrt{\frac{2 K}{1+v^{2}}} \phi^{\alpha / 2}(1-\phi)^{\beta / 2}
$$

If $\alpha$ were greater than 2 the Cauchy-Lipschitz theorem would apply using the boundary condition $\phi(0)=0$ or $\phi(L)=0$ and the unique solution would be $z=\phi=0$. Now because we supposed $\alpha \in(1 ; 2)$, we can have a local nonuniqueness result.

Let's build a solution different from 0 . First we can consider that $\phi$ is equal to zero in a neighborhood of 0 e.g. $\forall s \in[0, \eta]$. Now for $s>\eta$, we can integrate the ODE and define $\phi(s)$ implicitly by:

$$
\int_{0}^{\phi(s)} w^{-\alpha / 2}(1-w)^{-\beta / 2} d w=\sqrt{\frac{2 K}{1+v^{2}}}(s-\eta)
$$

This definition holds as long as $\phi(s) \leq 1$, i.e. as long as $s \leq M$ where $M$ is defined as $B(1-\alpha / 2,1-\beta / 2)=\sqrt{\frac{2 K}{1+v^{2}}}(M-\eta)(B$ stands for the beta function).

Now, for $s>M$, we build the solution in the same way and we can do so because $\beta \in(1 ; 2)$. We define implicitly $\phi(s)$ by:

$$
\int_{\phi(s)}^{1} w^{-\alpha / 2}(1-w)^{-\beta / 2} d w=\sqrt{\frac{2 K}{1+v^{2}}}(s-M)
$$

as long as $\phi(s)$ remains positive. This happens in $s=M^{\prime}$ where $B(1-\alpha / 2,1-$ $\beta / 2)=\sqrt{\frac{2 K}{1+v^{2}}}\left(M^{\prime}-M\right)$.

Now, $\phi$ is supposed to be 0 for $s \geq M^{\prime}$.

We have built a differentiable function $\phi$ but we need to check that $M^{\prime}$ can be smaller than $L$ for a sufficiently small $\eta$.

We have $2 B(1-\alpha / 2,1-\beta / 2)=\sqrt{\frac{2 K}{1+v^{2}}}\left(M^{\prime}-\eta\right)$. Hence $M^{\prime}$ can be smaller than $L$ if and only if there exists $\eta$ such that

$$
\eta<L-\sqrt{\frac{2\left(1+v^{2}\right)}{K}} B(1-\alpha / 2,1-\beta / 2)
$$

Such a positive $\eta$ exists if and only if $\sqrt{\frac{K}{2\left(1+v^{2}\right)}} L>B(1-\alpha / 2,1-\beta / 2)$ and this is equivalent to our condition thanks to the link between the functions $\Gamma$ and $B$.

We can represent a solution $\phi$ as described above (supporters do not keep standing before going down to the seated position. This solution is not unique in general for two reasons. The first one is obvious: $\eta$ in the preceding proof can be chosen in an interval. However, this non-uniqueness is only due to a translation 
invariance of the problem on the torus and is therefore meaningless. A second reason is that supporters may stand for a while before going back to the seated position.

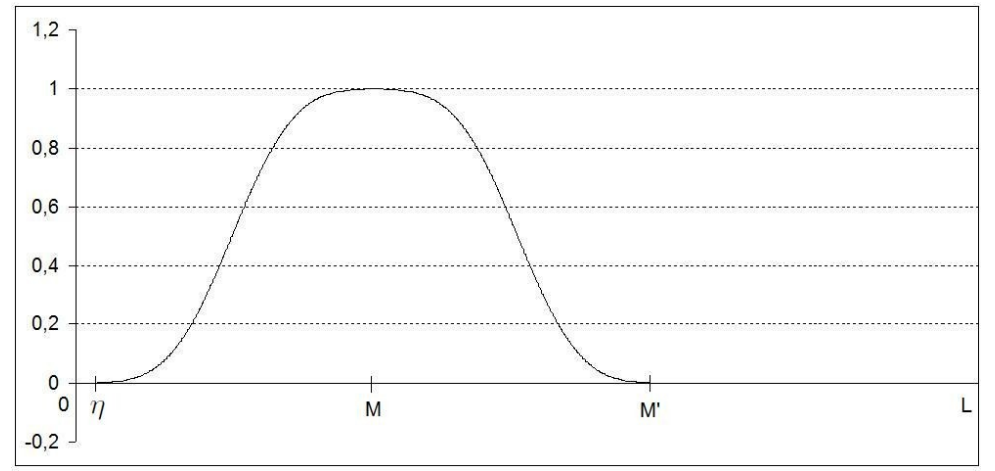

Figure 4: $\alpha=\beta=1.5$

\subsection{Mean field games versus descriptive models}

All the models developed to represent the Mexican wave assume that the supporters behave like automata: they carry out actions according to the context with possibly some memory of the preceding moments. This logic of automatic functioning is perfectly adequate for producing a Mexican wave-type crowd movement, and even for producing an equation of the dynamics that is the same as what we have written: if it has not already been done, we would be able to do so.

The difference between our model and a model based on automata agents (see for instance [19]), lies in the meaning given to the agents' actions. While automata produce actions dictated by the context, our agents produce the same actions as a consequence of a process of thinking about the behavior of other agents, the coherence of these behaviors, and the personal preferences in view of these behaviors. That this gives the same result as if agents were automata should not be cause for disappointment: the parsimony principle does not apply here; simply because agents behave as if they were automata is no reason for not giving another meaning to their actions. And not only for ethical reasons. Indeed, if one wishes to study the stability of the Mexican wave, and behaviors apart from equilibrium, it becomes necessary to return to the mechanism that has enabled the equation to be constructed. And hence, if the Mexican wave has been disturbed, if some rows of spectators have not moved for an exogenous reason, models based on automata generally predict erratic behavior in situations in which our mean field games agents, after a moment's thought, behave in such a way that the collective motion of the Mexican wave is re-established. Thus the meaning given to behaviors sheds light on what one would expect in 
the event of disturbance to various processes.

\section{A model of population distribution}

\section{$5.1 \quad$ Introduction}

Let's now consider a model of population distribution. This model is the archetype of a mean field games model in continuous time with a continuous state space. Many models can be derived from this one and most importantly the notion of stability introduced in what follows is arguably one of the most relevant one to deal with stability in forward/backward models such as intertemporal mean field games models. This stability notion called eductive stability turns out to be useful to circumvent the issues of the forward/backward structure, especially when it comes to find numerical solutions to mean field games PDEs.

In what follows we only present some aspects of the model. The readers may refer to [23] to go deeper into the different notions of stability or they may read [22] for an even more complete presentation with generalization to multipopulation issues.

\subsection{The framework}

We consider a large number of agents modeled as usual by a continuum. These agents have geographic, economic and social characteristics that we assume are represented by a finite number $n$ of values. A simple example is the position of an agent represented by his coordinates in space. Another example is that of a technology used by an agent. In short, we assume that the agents have characteristics denoted by $X \in \mathbb{R}^{n}$.

Each individual will have control over his characteristics, and we choose the case in which agents wish to resemble their peers. To resemble others, an agent has to move in the state $\mathbb{R}^{n}$. When an agent wants to make move of size $\alpha$ in the characteristics space (hereafter social space or state space) he will pay a cost of the quadratic form $\frac{|\alpha|^{2}}{2}$. Moreover, this control is not perfect, since we add Brownian noise. In mathematical terms, our problem is thus written (for an agent $i$ ):

$$
\begin{gathered}
\sup _{\left(\alpha_{s}\right)_{s \geq 0}, X_{0}^{i}=x} \mathbb{E}\left[\int_{0}^{\infty}\left(g\left(t, X_{t}^{i}, m_{t}\right)-\frac{\left|\alpha\left(s, X_{s}^{i}\right)\right|^{2}}{2}\right) e^{-\rho s} d s\right] \\
d X_{t}^{i}=\alpha\left(t, X_{t}^{i}\right) d t+\sigma d W_{t}^{i}
\end{gathered}
$$

where $m$ is the distribution of agents in the social space and where the function $g$ will model the will to resemblance depending on the type of problem. Various specifications for $g$ will produce our results: 


$$
\begin{gathered}
g(t, x, m)=-\beta\left(x-\int y m(t, y) d y\right)^{2} \\
g(t, x, m)=-\beta \int(x-y)^{2} m(t, y) d y \\
g(t, x, m)=\ln (m(t, x))
\end{gathered}
$$

To simplify the exposition and to stick to the papers cited in the introduction, we consider the logarithmic case where $g$ is a local function of $m$.

The control problem is re-written in differential way. We obtain the PDEs which are at the heart of mean field games theory:

$$
\begin{aligned}
\text { (HJB) } \quad \partial_{t} u+\frac{\sigma^{2}}{2} \Delta u+\frac{1}{2}|\nabla u|^{2}-\rho u & =-\ln (m) \\
\text { (Kolmogorov) } \quad \partial_{t} m+\nabla \cdot(m \nabla u) & =\frac{\sigma^{2}}{2} \Delta m
\end{aligned}
$$

and in this case the optimal control is written $\alpha\left(t, X_{t}\right)=\nabla u\left(t, X_{t}\right)$.

What is fundamental in this PDE system is the forward/backward dimension. The Hamilton Jacobi Bellman equation is backward like all Bellman equations (in finite time, there would be a final condition of type $\left.u(T, x)=u_{T}(x)\right)$ - this can also be seen in the diffusion part of the equation, which is a backward heat equation. Conversely, the transport equation is forward and transport an initial distribution $m(0, x)$ according to agents' optimal choices. We clearly see the emergence of agents' reasoning in this forward/backward aspect. They assume a dynamic for $m$ and optimize as a result to get the optimal control $\nabla u$. The behavior we obtained transports the distribution of agents. The coherence is finally found if we assume that the expectations are rational (and hence perfect) on the distribution $m$. This is the usual reasoning in mean field games.

\subsection{Stationary solutions}

We are interested firstly in stationary solutions. The framework of quadratic costs and logarithmic utility allows us to have explicit quadratic solutions for $u$ and Gaussian solutions for $m$, as in the following result:

Proposition 5.1 (Gaussian solutions). Suppose that $\rho<\frac{2}{\sigma^{2}}$.

There exist three constants, $s^{2}>0, \eta>0$ and $\omega$ such that $\forall \mu \in \mathbb{R}^{n}$, if $m$ is the probability distribution function associated to a gaussian variable $\mathcal{N}\left(\mu, s^{2} I_{n}\right)$ and $u(x)=-\eta|x-\mu|^{2}+\omega$, then $(u, m)$ is a solution of our problem.

These three constants are given by:

$$
\text { - } s^{2}=\frac{\sigma^{4}}{4-2 \rho \sigma^{2}}
$$


- $\eta=\frac{1}{\sigma^{2}}-\frac{\rho}{2}=\frac{\sigma^{2}}{4 s^{2}}$

- $\omega=-\frac{1}{\rho}\left[\eta n \sigma^{2}-\frac{n}{2} \ln \left(\frac{2 \eta}{\pi \sigma^{2}}\right)\right]$

Proof:

First, let's note that the stationary equation for $m$ (the Kolmogorov equation) can be rewritten as:

$$
\nabla \cdot\left(m \nabla u-\frac{\sigma^{2}}{2} \nabla m\right)=0
$$

Hence, we can restrict ourselves to solutions of:

$$
m \nabla u=\frac{\sigma^{2}}{2} \nabla m
$$

Consequently, we just need to solve the Hamilton Jacobi Bellman equation if we replace $m$ by $K \exp \left(\frac{2}{\sigma^{2}} u\right)$ where $K$ is chosen to ensure that $m$ is indeed a probability distribution function.

We are looking for a solution for $u$ of the form:

$$
u(x)=-\eta|x-\mu|^{2}+\omega
$$

If we put this form in the Hamilton Jacobi Bellman equation we get:

$$
2 \eta^{2}|x-\mu|^{2}+\rho \eta|x-\mu|^{2}-\rho \omega-\eta n \sigma^{2}=-\ln (K)+\frac{2 \eta|x-\mu|^{2}}{\sigma^{2}}-\frac{2 \omega}{\sigma^{2}}
$$

A first condition for this to be true is:

$$
\begin{aligned}
& 2 \eta^{2}+\rho \eta=\frac{2 \eta}{\sigma^{2}} \\
& \Longleftrightarrow \eta=\frac{1}{\sigma^{2}}-\frac{\rho}{2}
\end{aligned}
$$

A second condition, to find $\omega$, is related to the fact that $m$ is a probability distribution function. This clearly requires $\eta$ to be positive but this is guaranteed by the hypothesis $\rho \sigma^{2}<2$. This also implies:

$$
\begin{gathered}
K \exp \left(\frac{2 \omega}{\sigma^{2}}\right) \int_{\mathbb{R}^{n}} \exp \left(\frac{-2 \eta}{\sigma^{2}}|x-\mu|^{2}\right)=K \exp \left(\frac{2 \omega}{\sigma^{2}}\right)\left(\frac{\pi \sigma^{2}}{2 \eta}\right)^{\frac{n}{2}}=1 \\
\Rightarrow \rho \omega+\eta n \sigma^{2}=\frac{n}{2} \ln \left(\frac{2 \eta}{\pi \sigma^{2}}\right)
\end{gathered}
$$

and this last equation gives $\omega$.

From this solution for $u$ we can find a solution for $m$. We indeed know that $m$ is a probability distribution function and that $m$ is given by

$$
m(x)=K \exp \left(\frac{2 u(x)}{\sigma^{2}}\right)
$$


As a consequence, $m$ is the probability distribution function of an n-dimensional gaussian random variable with variance equal to $s^{2} I_{n}$ where $s^{2}=\frac{\sigma^{2}}{4 \eta}$ i.e. $s^{2}=\frac{\sigma^{4}}{4-2 \rho \sigma^{2}}$.

A priori, nothing guaranteed that a solution exists insofar as the cases usually well treated (see [36]) correspond most often to a decreasing function $g$ and not, as here, to an increasing one. On the other hand nothing shows there is a uniqueness result. First, there is invariance by translation and we must therefore localize the problem in order to address this question. This localization is done simply by replacing $\ln (m(t, x))$ by $\ln (m(t, x))-\delta x^{2}(\delta>0)$ and we obtain the same type of results. Even when localized, we do not guarantee uniqueness (though, localization will be helpful for other purposes). Although we do not prove uniqueness, we are nevertheless interested in the problem of the stability of solutions. Since we have a stationary result, a first step towards studying the dynamics is to study stability.

\subsection{Stability results}

\subsubsection{Two notions of stability}

Two types of stability are relevant to our problem. We will call the first one "physical stability" and the second one "eductive stability". The physical stability concept might look more standard to the reader. The second, the eductive stability, refers to many papers by Roger Guesnerie and other authors (e.g. [26]) on stability in a rational expectation economic context. These papers inspired the mean field games eductive stability concept.

If we consider a problem on $[0, T]$ with conditions equal to the stationary solutions on each side $\left(u(T, \cdot)=u^{*}\right.$ and $m(0, \cdot)=m^{*}$ given), we can look at what happens (as $T \rightarrow \infty$ ) when we disturb the boundary conditions $\left(u^{*}, m^{*}\right)$. The stability associated with this perturbation in 0 for $m$ and in $T$ for $u$ is the physical stability and we refer to [23] for a complete study.

A second possibility is to add a variable $\theta$ (vitual time) and to consider a different, purely forward, dynamic system, whose stationary equilibrium is the same as the one we are looking for. If there is convergence (when $\theta \rightarrow \infty$ ) in this new dynamic system where we reverse the time in the backward equation by imposing arbitrary conditions in $\theta=0$, then we shall call this eductive stability.

In what follows, we focus on eductive stability, and more precisely on local eductive stability, because it helps a lot to develop and justify numerical methods.

\subsubsection{Eductive stability}

The physical stability, briefly described above, is intrinsically linked to the forward/backward structure of the equations. 
Here, we want to circumvent this forward/backward structure and we introduce a virtual time $\theta$ that will be purely forward in the sense that we consider the following new system of $\mathrm{PDEs}^{12}$ :

$$
\begin{gathered}
\partial_{\theta} u=\frac{\sigma^{2}}{2} u^{\prime \prime}+\frac{1}{2} u^{\prime 2}-\rho u+\ln (m) \\
\partial_{\theta} m=\frac{\sigma^{2}}{2} m^{\prime \prime}-\left(m u^{\prime}\right)^{\prime}
\end{gathered}
$$

Let's consider two "initial guesses" $(u(\theta=0, x)$ and $m(\theta=0, x))$ that are not too far from the stationary equilibrium $\left(u^{*}, m^{*}\right)$ associated to $\mu=0$, as defined in Proposition 5.1:

$$
\begin{gathered}
m(0, x)=m^{*}(x)(1+\varepsilon \psi(0, x)) \\
u(0, x)=u^{*}(x)+\varepsilon \phi(0, x)
\end{gathered}
$$

We are going to linearize these equations. After easy computations we obtain the following linear PDEs:

$$
\begin{gathered}
\partial_{\theta} \phi=\frac{\sigma^{2}}{2} \phi^{\prime \prime}-2 \eta x \phi^{\prime}-\rho \phi+\psi \\
\partial_{\theta} \psi=\frac{\sigma^{2}}{2} \psi^{\prime \prime}-2 \eta x \psi^{\prime}-\phi^{\prime \prime}+\frac{x}{s^{2}} \phi^{\prime}
\end{gathered}
$$

A more convenient way to write these linearized PDEs is to introduce the operator $\mathcal{L}: f \mapsto \mathcal{L} f=-\frac{\sigma^{2}}{2} f^{\prime \prime}+2 \eta x f^{\prime}$ and we get the following equations for the couple $(\phi, \psi)$ :

\section{Proposition 5.2.}

$$
\begin{aligned}
\partial_{\theta} \phi & =-\mathcal{L} \phi-\rho \phi+\psi \\
\partial_{\theta} \psi & =-\mathcal{L} \psi+\frac{2}{\sigma^{2}} \mathcal{L} \phi
\end{aligned}
$$

\section{Proof:}

It simply is a consequence of the link between the variables, namely $s^{2}=\frac{\sigma^{2}}{4 \eta}$.

Now, we are going to use the properties of the operator $\mathcal{L}$ we have just introduced. To do that we need to use some properties of the Hermite polynomials associated to the space $L^{2}\left(m^{*}(x) d x\right)$ (see [1] for more details).

Proposition 5.3 (Hermite polynomials). We define the $n^{\text {th }}$ Hermite polynomial of $L^{2}\left(m^{*}(x) d x\right)$ by:

$$
H_{n}(x)=s^{n} \frac{1}{\sqrt{n !}}(-1)^{n} \exp \left(\frac{x^{2}}{2 s^{2}}\right) \frac{d^{n}}{d x^{n}} \exp \left(-\frac{x^{2}}{2 s^{2}}\right)
$$

\footnotetext{
${ }^{12}$ We consider the problem in dimension 1 for the sake of simplicity but the problem in general is the same
} 
The polynomials $\left(H_{n}\right)_{n}$ form an orthonormal basis of the Hilbert space $L^{2}\left(m^{*}(x) d x\right)$.

The Hermite polynomials $H_{n}$ are eigenvectors of $\mathcal{L}$ and:

$$
\mathcal{L} H_{n}=2 \eta n H_{n}
$$

To study the linearized equations, we are going to consider the space $L^{2}\left(m^{*}(x) d x\right)$ and consider a decomposition on the Hermite polynomials basis. Because the problem is purely forward in $\theta$ we need to have, for each coordinate, two negative eigenvalues.

To this purpose, let's introduce the matrices $\left(B_{n}\right)_{n}$ :

$$
B_{n}=\left(\begin{array}{cc}
-(\rho+2 \eta n) & 1 \\
\frac{n}{s^{2}} & -2 \eta n
\end{array}\right)
$$

Lemma 5.4 (Eigenvalues of $B_{n}$ ). Let's consider $n \geq 2$.

The eigenvalues $\xi_{n}^{1}<\xi_{n}^{2}$ of $B_{n}$ are both negative with:

$$
\xi_{n}^{1,2}=\frac{1}{2}\left[-\rho-4 \eta n \pm \sqrt{\rho^{2}+\frac{4 n}{s^{2}}}\right]
$$

Proposition 5.5. Let's suppose that the initial conditions $\phi(0, \cdot)$ and $\psi(0, \cdot)$ are in the Hilbert space $H=L^{2}\left(m^{*}(x) d x\right)$.

Let's consider for $n \geq 2$ the functions $\left(\begin{array}{c}\phi_{n} \\ \psi_{n}\end{array}\right)$ that verify:

$$
\left(\begin{array}{c}
\partial_{\theta} \phi_{n} \\
\partial_{\theta} \psi_{n}
\end{array}\right)=B_{n}\left(\begin{array}{c}
\phi_{n} \\
\psi_{n}
\end{array}\right)
$$

with $\phi_{n}(0)$ equal to $\phi(0, \cdot)_{n}=<H_{n}, \phi_{n}(0)>$ and $\psi_{n}(0)$ equal to $\psi(0, \cdot)_{n}=<$ $H_{n}, \psi_{n}(0)>$.

We have for a fixed $\theta$ and as $n$ tends to $\infty$ :

$$
\begin{gathered}
\phi_{n}(\theta)=\mathcal{O}\left(\left|\phi_{n}(0)\right| e^{\xi_{n}^{2} \theta}\right) \\
\psi_{n}(\theta)=\mathcal{O}\left(\sqrt{n}\left|\phi_{n}(0)\right| e^{\xi_{n}^{2} \theta}\right)
\end{gathered}
$$

In particular,

$$
\forall \theta>0, \forall k \in \mathbb{N},\left(n^{k} \phi_{n}(\theta)\right)_{n} \in l^{1}\left(\subset l^{2}\right),\left(n^{k} \psi_{n}(\theta)\right)_{n} \in l^{1}\left(\subset l^{2}\right)
$$

\section{Proof:}

After straightforward algebraic manipulations, we get:

$$
\left(\begin{array}{c}
\phi_{n}(\theta) \\
\psi_{n}(\theta)
\end{array}\right)=A_{n} e^{\xi_{n}^{1} \theta}\left(\begin{array}{c}
1 \\
a_{n}
\end{array}\right)+B_{n} e^{\xi_{n}^{2} \theta}\left(\begin{array}{c}
1 \\
b_{n}
\end{array}\right)
$$


where:

$$
a_{n}=\rho+2 \eta n+\xi_{n}^{1}, \quad b_{n}=\rho+2 \eta n+\xi_{n}^{2}
$$

Now, to find the two constants we need to use the conditions on $\phi_{n}(0)$ and $\psi_{n}(0)$ :

$$
\left\{\begin{array}{c}
\phi_{n}(0)=A_{n}+B_{n} \\
\psi_{n}(0)=a_{n} A_{n}+b_{n} B_{n}
\end{array}\right.
$$

Hence:

$$
\left\{\begin{array}{l}
A_{n}=\frac{b_{n} \phi_{n}(0)-\psi_{n}(0)}{b_{n}-a_{n}} \\
B_{n}=\frac{a_{n} \phi_{n}(0)-\psi_{n}(0)}{a_{n}-b_{n}}
\end{array}\right.
$$

Using the fact that $a_{n} \sim-\frac{\sqrt{\eta}}{\sigma} \sqrt{n}$ and $b_{n} \sim \frac{\sqrt{\eta}}{\sigma} \sqrt{n}$ we can deduce the asymptotic behavior of the constants as $n$ goes to infinity.

$$
A_{n} \sim_{n \rightarrow \infty} \frac{\phi_{n}(0)}{2}, \quad B_{n} \sim_{n \rightarrow \infty} \frac{\phi_{n}(0)}{2}
$$

Hence, since $\xi_{n}^{1}<\xi_{n}^{2}$,

$$
\begin{gathered}
\phi_{n}(\theta)=\mathcal{O}\left(\left|\phi_{n}(0)\right| e^{\xi_{n}^{2} \theta}\right) \\
\psi_{n}(\theta)=\mathcal{O}\left(\sqrt{n}\left|\phi_{n}(0)\right| e^{\xi_{n}^{2} \theta}\right)
\end{gathered}
$$

These two estimations prove the results.

These estimations show that the solutions will be far more regular than the initial conditions.

Proposition 5.6 (Resolution of the linearized PDEs). Suppose that:

- The initial conditions $\phi(0, \cdot)$ and $\psi(0, \cdot)$ are in the Hilbert space $H=$ $L^{2}\left(m^{*}(x) d x\right)$.

- $\int \psi(0, x) m^{*}(x) d x=0$ (this is guaranteed if the initial guess for $m$ is a probability distribution function)

- $\int x \phi(0, x) m^{*}(x) d x=0$ (this is guaranteed if the initial guess is even)

- $\int x \psi(0, x) m^{*}(x) d x=0$ (this is guaranteed if the initial guess is even)

Let's define $\left(\phi_{n}\right)_{n}$ and $\left(\psi_{n}\right)_{n}$ by:

- $\phi_{0}(\theta)=\phi_{0}(0) e^{-\rho \theta}$ and $\psi_{0}(\theta)=0$.

- $\phi_{1}(\theta)=\psi_{1}(\theta)=0$.

- $\forall n \geq 2, \phi_{n}$ and $\psi_{n}$ defined as in the preceding proposition. 
Then $\phi(\theta, x)=\sum_{n=0}^{\infty} \phi_{n}(\theta) H_{n}(x)$ and $\psi(\theta, x)=\sum_{n=0}^{\infty} \psi_{n}(\theta) H_{n}(x)$ are well defined in $H$, are in $C^{\infty}\left(\mathbb{R}_{+}^{*}\right)$, are solutions of the PDEs and verify the initial conditions.

\section{Proof:}

First of all, the above proposition ensures that for $\theta>0$ the two functions $\phi$ and $\psi$ are well defined, in $C^{\infty}$, and that we can differentiate formally the expressions. Then, the first three conditions can be translated as $\psi_{0}(0, \cdot)=0$, $\phi_{1}(0, \cdot)=0$ and $\psi_{1}(0, \cdot)=0$ and so the conditions at time 0 is verified.

The fact that the PDEs are verified is due to the definition of $\phi_{n}$ and $\psi_{n}$ and also to the fact that we can differentiate under the sum sign because of the estimates of the preceding proposition.

Proposition 5.7 (Local eductive stability). Suppose that:

- The initial guesses $\phi(0, \cdot)$ and $\psi(0, \cdot)$ are in the Hilbert space $H=L^{2}\left(m^{*}(x) d x\right)$.

- $\int \psi(0, x) m^{*}(x) d x=0$ (this is guaranteed if the initial guess for $m$ is a probability distribution function)

- $\int x \phi(0, x) m^{*}(x) d x=0$ (this is guaranteed if the initial guess is even)

- $\int x \psi(0, x) m^{*}(x) d x=0$ (this is guaranteed if the initial guess is even)

Then the solution $(\phi, \psi)$ of the PDEs converges in the sense that:

$$
\lim _{\theta \rightarrow \infty}\|\phi(\theta, \cdot)\|_{L^{2}\left(m^{*}(x) d x\right)}=0 \quad \lim _{\theta \rightarrow \infty}\|\psi(\theta, \cdot)\|_{L^{2}\left(m^{*}(x) d x\right)}=0
$$

\section{Proof:}

We basically want to show that:

$$
\sum_{n=0}^{+\infty}\left|\phi_{n}(\theta)\right|^{2} \rightarrow_{\theta \rightarrow+\infty} 0, \quad \sum_{n=0}^{+\infty}\left|\psi_{n}(\theta)\right|^{2} \rightarrow_{\theta \rightarrow+\infty} 0
$$

This is actually a pure consequence of the estimates proved earlier and of the Lebesgue's dominated convergence theorem.

These stability results are interesting but the symmetry conditions to obtain them may seem cumbersome. Indeed, when it comes to apply this kind of methodology to find stationary solutions, we clearly need a result that is less sensitive to initial conditions. A good way to proceed is to consider the case introduced at the beginning where there is no translation invariance, that is the localized case in which $\delta>0$. 


\subsubsection{Eductive stability in the localized case}

In the proof of the eductive stability, there was a need to impose symmetry conditions on the initial guesses. These conditions were necessary to ensure stability because $B_{1}$ was singular. If one wants to have stability results for more general initial guesses, the intuitive idea is to break the translation invariance of the problem.

Interestingly, we introduced localization earlier. This localization idea can be used once again, to have more general stability results. If we center the problem around 0 as before, we can see that the only relevant difference between the original problem and the problem with an additional term $-\delta x^{2}$, that localizes the problem around 0 , is the positive constant $\eta$ that depends on $\delta$ according to the equation:

$$
2 \eta^{2}-\eta\left(\frac{2}{\sigma^{2}}-\rho\right)=\delta
$$

Now, in this context we can prove that the eigenvalues of $B_{n}$ are both negative for $n \geq 1$ (remember that we needed $n$ to be larger than 2 to have these properties in the case where $\delta=0$ ).

This result can be used to prove general stability results when $\delta>0$. It is indeed straightforward that all our stability results can be rewritten exactly the same if one replaces the conditions

$$
\left\{\begin{array}{l}
\int x \psi(0, x) m^{*}(x) d x=0 \\
\int x \phi(0, x) m^{*}(x) d x=0
\end{array}\right.
$$

by $\delta>0$.

Thus, in this localized context, (and up to a linear approximation) if we start from a couple $(u, m)$ close to a stationary equilibrium, there will be convergence toward stationary equilibrium as $\theta \rightarrow \infty$ when using the purely forward PDEs system. Numerically, this is very interesting and the eductive methods give very good results, both for finding stationary equilibrium and for generalizing the approach for seeking dynamic equilibrium (see [23]).

\subsection{Numerical methods}

The forward/backward structure of mean field games is quite an issue when it comes to find numerical solutions. One can try to find a fixed point $(u, m)$ solving alternatively the backward equation and the forward equation but there is a priori no guarantee that a solution can be found in this way. The eductive stability property proved earlier, however, can be adapted to design a numerical method. Other authors have developed several methods and the interested author may for instance see [3].

We are going to present our methods to find stationary solutions. Interestingly, if one replaces the Laplace operator by heat operators (forward or backward, 
depending on the context), the numerical recipes presented below still work to find dynamical solutions (see $[23,22]$ ).

\subsubsection{Stationary equilibrium}

First, let's recall the two equations that characterize a stationary equilibrium:

$$
\frac{\sigma^{2}}{2} \Delta u+\frac{1}{2}|\nabla u|^{2}-\rho u=-g(x, m)
$$

$$
\text { (Kolmogorov) } \quad \nabla \cdot(m \nabla u)=\frac{\sigma^{2}}{2} \Delta m
$$

where, now, $g$ is not anymore supposed to be the logarithm function.

The Hamilton Jacobi Bellman equation can be simplified using the change of variable $^{13} \beta=\exp \left(\frac{u}{\sigma^{2}}\right)$ and we obtain:

$$
\begin{aligned}
& \text { (HJB), } \quad \frac{\sigma^{2}}{2} \Delta \beta=\beta\left[\rho \ln (\beta)-\frac{1}{\sigma^{2}} g(x, m)\right] \\
& \text { (Kolmogorov), } \quad \nabla \cdot\left[\sigma^{2}\left(m \frac{\nabla \beta}{\beta}\right)\right]=\frac{\sigma^{2}}{2} \Delta m
\end{aligned}
$$

The two equations (HJB)' and (Kolmogorov)' can be written in a more practical way for numerical resolutions by "inverting" the $\Delta$ operators. This can be done in the Kolmogorov equation by restricting the Laplace operator to probability distribution functions and we obtain:

$$
\text { (Kolmogorov) }, \quad-m+\left[\frac{\sigma^{2}}{2} \Delta\right]^{-1}\left(\sigma^{2} \nabla \cdot\left(m \frac{\nabla \beta}{\beta}\right)\right)=0
$$

This cannot be done in the case of the Hamilton Jacobi Bellman equation but we can invert an operator like $\frac{\sigma^{2}}{2} \Delta-\epsilon I d$ for any $\epsilon>0$. This gives:

$$
(\mathrm{HJB}), \quad-\beta+\left[\frac{\sigma^{2}}{2} \Delta-\epsilon I d\right]^{-1}\left(\beta\left[\rho \ln (\beta)-\frac{1}{\sigma^{2}} g(x, m)-\epsilon\right]\right)=0
$$

Using these equations we can consider the ideas of eductive stability and try to obtain solutions by solving the following equations where we introduce the virtual time $\theta$ :

\footnotetext{
${ }^{13}$ This change of variable, combined with the change of variable $\alpha=m \exp \left(-\frac{u}{\sigma^{2}}\right)$, simplifies the PDEs. Easy calculations give in fact that the equations in $(u, m)$ become:

$$
\begin{gathered}
\partial_{t} \beta+\frac{\sigma^{2}}{2} \Delta \beta=\beta h(\alpha, \beta) \\
\partial_{t} \alpha-\frac{\sigma^{2}}{2} \Delta \alpha=-\alpha h(\alpha, \beta)
\end{gathered}
$$

where $h$ simply is $h(\alpha, \beta)=\rho \ln (\beta)-\frac{1}{\sigma^{2}} g(\alpha \beta)$
} 


$$
\left\{\begin{array}{l}
\partial_{\theta} m=-m+\left[\frac{\sigma^{2}}{2} \Delta\right]^{-1}\left(\sigma^{2} \nabla \cdot\left(m \frac{\nabla \beta}{\beta}\right)\right) \\
\partial_{\theta} \beta=-\beta+\left[\frac{\sigma^{2}}{2} \Delta-\epsilon I d\right]^{-1}\left(\beta\left[\rho \ln (\beta)-\frac{1}{\sigma^{2}} g(x, m)-\epsilon\right]\right)
\end{array}\right.
$$

Numerically these equations are quite easy to solve using Fourier series. An example is shown below where $g(x, m)=\sqrt{m}-\delta x^{2}$ with $\sigma^{2}=0.4, \rho=0.4$, $\delta=0.5$ on the domain $[-1,1]$ (we took $\left.\epsilon=\frac{\rho}{3}\right)$.

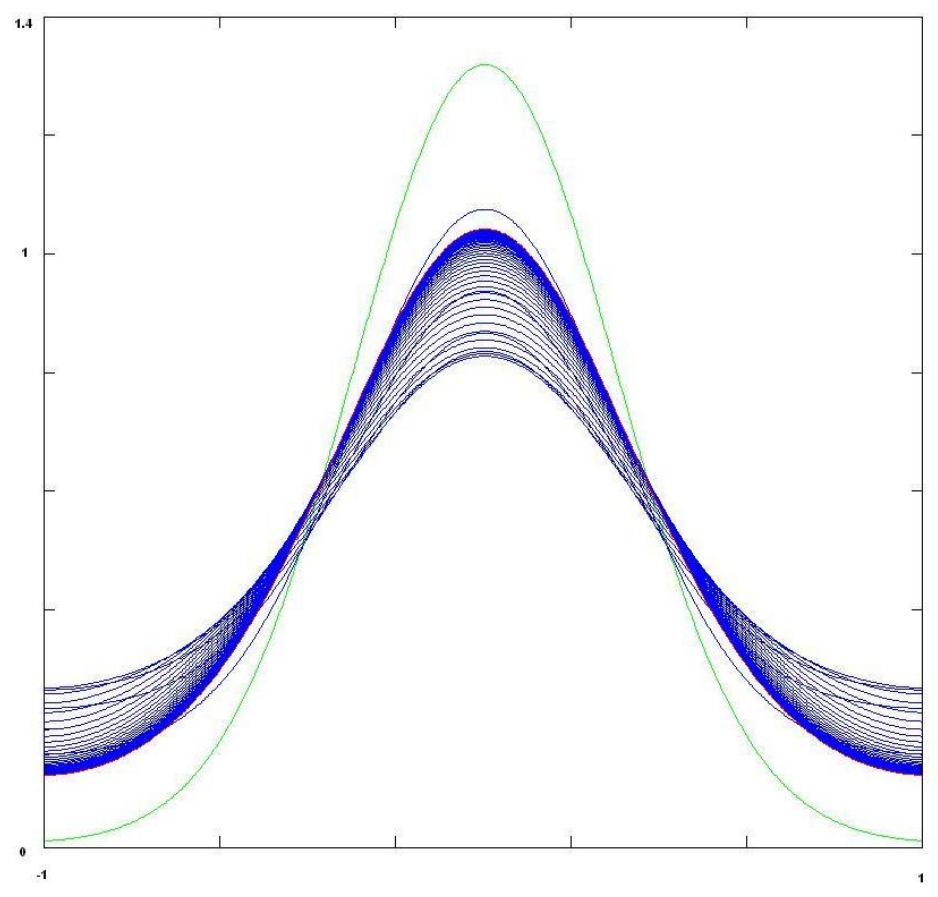

Figure 5: Initial guess $\simeq \mathcal{N}(0,0.3)$. Solution after 8000 iterations with $d \theta \simeq 0.01$ (an iteration is drawn every 40 iterations).

We see that after a certain number of steps in $\theta$, the distribution $m(\theta, \cdot)$ converges towards a limit distribution $m$ that is a good candidate for being a stationary equilibrium.

\subsubsection{Generalizations}

This method works really well in practice for stationary solutions. In addition to be fast and effective, the eductive algorithm (as we term it) can be generalized to find not only stationary solutions but dynamical solutions of the mean field game PDEs. In short, the idea is simply to invert the heat operators instead of Laplace operators before introducing the virtual time $\theta$. This is done in $[23,22]$. 


\section{$6 \quad$ Asset managers and ranking effect}

\subsection{Introduction}

When someone entrusts his saving to an asset manager, he does so according to his risk profile, i.e. he will try and find an asset manager whose management is as close as possible, in terms of return/risk for example, to what would be his own management strategy were it not delegated. However, the asset manager to whom he entrusts his savings does not have the sole aim of satisfying his current customers. He may wish to increase the number of his customers and therefore the assets under management or, as an individual, perform better in order to increase his bonus.

We offer a model which, starting off from the classic Markowitz model (see [41]) or the CARA-Gaussian model, adds a ranking dimension among the asset managers: each asset manager will want to optimize, over and above his usual criterion, a function that depends on his ranking (in terms of return) among all the asset managers.

\subsection{The model}

Our model therefore considers a continuum of asset managers who at time 0 have the same unitary amount to manage. These managers will invest in riskfree and risky assets in creating their portfolio. A proportion $\theta$ of their portfolio will be invested in risky assets and a proportion $1-\theta$ in risk-free assets with return $r$. The risky assets have a return which we denote $r+\tilde{\epsilon}$, where $\tilde{\epsilon}$ is a random variable that we will assume is distributed normally, with the mean and variance still be to specified.

To build their portfolio, managers will optimize a criterion of the following form:

$$
\mathbb{E}[u(X)+\beta \tilde{C}]
$$

where:

- $u(x)=-\exp (-\lambda x)$ is a CARA utility function.

- $X=1+r+\theta \tilde{\epsilon}$ is the fund value at date 1 .

- $\beta$ measures the relative importance of the additional criterion of competition among managers.

- $\tilde{C}$ is the random variable representing the ranking. This variable $\tilde{C}$ has values in $[0,1]$, with 0 corresponding to the worst performance and 1 to the best performance obtained by a manager.

It now remains to specify how managers differ. If they all have the same amount to invest, they nevertheless have different beliefs as regards the return on the 
risky asset, i.e. in relation to the variable $\tilde{\epsilon}$. These beliefs will concern the mean of $\tilde{\epsilon}$ (we assume that there is agreement on volatility), such that an agent will be of type $\epsilon$ if he thinks that $\tilde{\epsilon} \sim \mathcal{N}\left(\epsilon, \sigma^{2}\right)$. We will assume in what follows that the beliefs $\epsilon$ are distributed according to a probability distribution function $f$ (even, for example).

\subsection{Resolution}

To solve this problem, let us consider an agent of type $\epsilon$. The proportion $\theta$ of his portfolio placed in the risky asset is given by the following optimality condition:

Proposition 6.1 $\left(F O C_{\epsilon}\right)$.

$\left(F O C_{\epsilon}\right) \quad-\lambda^{2} \sigma^{2}\left(\theta-\frac{\epsilon}{\lambda \sigma^{2}}\right) \exp \left(-\lambda(1+r)-\lambda \theta \epsilon+\frac{1}{2} \lambda^{2} \theta^{2} \sigma^{2}\right)+\beta m(\theta) C(\epsilon)=0$

where $m$ is the distribution of the $\theta$ 's in the population at equilibrium and where $C(\cdot)=2\left[N(\dot{\bar{\sigma}})-\frac{1}{2}\right], N$ being the cumulative distribution function of a normal variable $\mathcal{N}(0,1)$.

\section{Proof:}

The asset manager maximizes:

$$
\mathbb{E}_{\epsilon}[u(1+r+\theta \tilde{\epsilon})+\beta \tilde{C}]
$$

It's easy to see that $\tilde{C}=1_{\tilde{\epsilon}>0} M(\theta)+1_{\tilde{\epsilon} \leq 0}(1-M(\theta))$ where $M$ stands for the cumulative distribution function of the weights $\theta$.

Also,

$$
\begin{gathered}
\mathbb{E}_{\epsilon}[u(1+r+\theta \tilde{\epsilon})]=-\mathbb{E}_{\epsilon}[\exp (-\lambda(1+r+\theta \tilde{\epsilon}))] \\
=-\exp \left(-\lambda(1+r+\theta \epsilon)+\frac{1}{2} \lambda^{2} \theta^{2} \sigma^{2}\right)
\end{gathered}
$$

Hence, the optimal $\theta$ is given by the argmax of:

$$
-\exp \left(-\lambda(1+r+\theta \epsilon)+\frac{1}{2} \lambda^{2} \theta^{2} \sigma^{2}\right)+\beta \mathbb{E}_{\epsilon}\left[1_{\tilde{\epsilon}>0} M(\theta)+1_{\tilde{\epsilon} \leq 0}(1-M(\theta))\right]
$$

Let's differentiate the above equation. We get the first order condition for an $\epsilon$-type asset manager:

$$
-\lambda^{2} \sigma^{2}\left(\theta-\frac{\epsilon}{\lambda \sigma^{2}}\right) \exp \left(-\lambda(1+r)-\lambda \theta \epsilon+\frac{1}{2} \lambda^{2} \theta^{2} \sigma^{2}\right)+\beta \mathbb{E}_{\epsilon}\left[1_{\tilde{\epsilon}>0}-1_{\tilde{\epsilon} \leq 0}\right] m(\theta)=0
$$


But,

$\mathbb{P}_{\epsilon}(\tilde{\epsilon}>0)-\mathbb{P}_{\epsilon}(\tilde{\epsilon} \leq 0)=2\left[\mathbb{P}_{\epsilon}(\tilde{\epsilon}>0)-\frac{1}{2}\right]=2\left[\mathbb{P}\left(\mathcal{N}(0,1)>-\frac{\epsilon}{\sigma}\right)-\frac{1}{2}\right]=C(\epsilon)$

Hence we get the result.

If we now use the fact that the solution $\epsilon \mapsto \theta(\epsilon)$ transport distribution $f$ toward distribution $m$, we see that the problem, once resolved, can be written in a differential way:

Proposition 6.2 (Differential equation for $\epsilon \mapsto \theta(\epsilon)$ ). Let's consider the function $\epsilon \mapsto \theta(\epsilon)$ that gives the optimal $\theta$ for each type. If $\theta$ is $C^{1}$ then it verifies the following differential equation:

$-\lambda^{2} \sigma^{2}\left(\theta-\frac{\epsilon}{\lambda \sigma^{2}}\right) \exp \left(-\lambda(1+r)-\lambda \theta \epsilon+\frac{1}{2} \lambda^{2} \theta^{2} \sigma^{2}\right) \frac{d \theta}{d \epsilon}+\beta f(\epsilon) C(\epsilon)=0$

Moreover, $\theta$ must verify $\theta(0)=0$.

\section{Proof:}

To go from the distribution $f$ of the types to the distribution $m$ of the $\theta$ 's, we need a coherence equation that is simply:

$$
m(\theta) \theta^{\prime}(\epsilon)=f(\epsilon)
$$

Now, if we take the different first order conditions $F O C_{\epsilon}$ and multiply by $\theta^{\prime}(\epsilon)$ we get the ODE we wanted to obtain.

Now, because $C(0)=0$, the equation $\left(F O C_{0}\right)$ is simply

$$
-\lambda^{2} \sigma^{2} \theta \exp \left(-\lambda(1+r)+\frac{1}{2} \lambda^{2} \theta^{2} \sigma^{2}\right)=0
$$

and the unique solution of this equation is $\theta=0$.

If we return to the Markowitz problem $(\beta=0)$, we see that the solution is simply given by $\epsilon \mapsto \theta_{0}(\epsilon)=\frac{\epsilon}{\lambda \sigma^{2}}$. Our problem with $\beta>0$ is therefore written:

$$
\theta^{\prime}(\epsilon)=\frac{\beta C(\epsilon) f(\epsilon)}{\lambda^{2} \sigma^{2} \exp \left(-\lambda(1+r+\theta(\epsilon) \epsilon)+\frac{1}{2} \lambda^{2} \sigma^{2} \theta(\epsilon)^{2}\right)} \frac{1}{\theta(\epsilon)-\theta_{0}(\epsilon)}, \quad \lim _{\epsilon \rightarrow 0} \theta(\epsilon)=0
$$

This is not a usual Cauchy problem since the condition in 0 is meaningful only at the limit. However, we should point out that the solution will be odd and that we can therefore restrict ourselves to $\epsilon>0$. Also, $\theta(\epsilon)$ must be increasing, 
which implies $\theta(\epsilon)>\theta_{0}(\epsilon)$ and hence greater risk-taking in our model than in the Markowitz model.

Now we can completely solve the problem and we get:

Proposition 6.3 (Existence and Uniqueness). There exists a unique function $\theta$ that verifies the equation $(*)$ with the two additional constraints:

- $\theta(\epsilon)>\theta_{0}(\epsilon)=\frac{\epsilon}{\lambda \sigma^{2}}$

- $\lim _{\epsilon \rightarrow 0} \theta(\epsilon)=0$

\section{Proof:}

Let's consider a solution $\theta$ of the problem and let's introduce the function $z$ defined by:

$$
z(\epsilon)=\frac{1}{2} \theta(\epsilon)^{2}-\theta_{0}(\epsilon) \theta(\epsilon)
$$

If we want to invert this equation and get $\theta$ as a function of $z$ then we get:

$$
\theta(\epsilon)=\theta_{0}(\epsilon) \pm \sqrt{\theta_{0}(\epsilon)^{2}+2 z(\epsilon)}
$$

but since $\theta(\epsilon)>\theta_{0}(\epsilon)$ we clearly can invert the equation and get:

$$
\theta(\epsilon)=\theta_{0}(\epsilon)+\sqrt{\theta_{0}(\epsilon)^{2}+2 z(\epsilon)}:=\Theta(\epsilon, z(\epsilon))
$$

Now, if we differentiate the equation that defines $z$ we have:

$$
\begin{gathered}
z^{\prime}(\epsilon)=\theta^{\prime}(\epsilon) \theta(\epsilon)-\theta_{0}(\epsilon) \theta^{\prime}(\epsilon)-\frac{1}{\lambda \sigma^{2}} \theta(\epsilon)=\theta^{\prime}(\epsilon)\left(\theta(\epsilon)-\theta_{0}(\epsilon)\right)-\frac{1}{\lambda \sigma^{2}} \theta(\epsilon) \\
\Rightarrow z^{\prime}(\epsilon)=\frac{\beta C(\epsilon) f(\epsilon)}{\lambda^{2} \sigma^{2} \exp \left(-\lambda(1+r+\theta(\epsilon) \epsilon)+\frac{1}{2} \lambda^{2} \sigma^{2} \theta(\epsilon)^{2}\right)}-\frac{1}{\lambda \sigma^{2}} \theta(\epsilon) \\
\Rightarrow z^{\prime}(\epsilon)=\frac{\beta C(\epsilon) f(\epsilon)}{\lambda^{2} \sigma^{2} \exp \left(-\lambda(1+r+\Theta(\epsilon, z(\epsilon)) \epsilon)+\frac{1}{2} \lambda^{2} \sigma^{2} \Theta(\epsilon, z(\epsilon))^{2}\right)}-\frac{1}{\lambda \sigma^{2}} \Theta(\epsilon, z(\epsilon))
\end{gathered}
$$

From Cauchy-Peano we know that there is a unique solution $z$ of this equation that verifies $z(0)=0$. This solution is defined in a neighborhood $V$ of 0 . From this we can prove that we can locally define, in the neighborhood $V, \theta$ by $\theta(\epsilon)=\theta_{0}(\epsilon)+\sqrt{\theta_{0}(\epsilon)^{2}+2 z(\epsilon)}$. This solution is unique because the differential equation that defines $z$ has a unique solution for $\epsilon>0$ (because of monotonicity). Hence, since there is no problem outside of 0 (i.e. the Cauchy-Lipschitz 
theorem can be applied directly) the uniqueness is proved.

Now, we want to prove that there exists a solution on the whole domain. For that let's consider the following ODE:

$$
z^{\prime}(\epsilon)=\frac{\beta C(\epsilon) f(\epsilon)}{\lambda^{2} \sigma^{2} \exp \left(-\lambda(1+r+\Theta(\epsilon, z(\epsilon)) \epsilon)+\frac{1}{2} \lambda^{2} \sigma^{2} \Theta(\epsilon, z(\epsilon))^{2}\right)}-\frac{1}{\lambda \sigma^{2}} \Theta(\epsilon, z(\epsilon))
$$

We know that there is a local solution $z$ (defined on a neighborhood $V$ of 0 ) satisfying this equation with $z(0)=0$.

If we define $\theta_{l o c}$ on $V$ (or more exactly on an open subset of $V$ that contains 0 , because it is not a priori defined on $V$ ) as:

$$
\theta_{l o c}(\epsilon)=\theta_{0}(\epsilon)+\sqrt{\theta_{0}(\epsilon)^{2}+2 z(\epsilon)}
$$

then, we have a local solution of the equation $(*)$ that satisfies the two additional conditions. Let's consider now $\hat{\epsilon}$ in $V$. We can apply the Cauchy Lipschitz theorem to the equation $(*)$ with the Cauchy condition $\theta(\hat{\epsilon})=\theta_{\text {loc }}(\hat{\epsilon})$ on the domain $\left\{(\epsilon, \theta) / \epsilon>0, \theta>\theta_{0}(\epsilon)\right\}$ and consider $\theta$ the maximal solution of the problem. This maximal solution clearly satisfies $\lim _{\epsilon \rightarrow 0} \theta(\epsilon)=0$. We want to show that there is in fact no upper bound for the maximal domain.

Suppose there is such an upper bound $\bar{\epsilon}$. Since $\theta$ is increasing, we have either:

$$
\lim _{\epsilon \rightarrow \bar{\epsilon}} \theta(\epsilon)=+\infty
$$

or

$$
\lim _{\epsilon \rightarrow \bar{\epsilon}} \theta(\epsilon)=\theta_{0}(\bar{\epsilon})
$$

We are going to show that these two cases are impossible.

Suppose first that $\lim _{\epsilon \rightarrow \bar{\epsilon}} \theta(\epsilon)=+\infty$. Then, we can suppose there exists an interval $(\underline{\epsilon}, \bar{\epsilon})$ such that $\forall \epsilon \in(\underline{\epsilon}, \bar{\epsilon}), \theta(\epsilon)>\theta_{0}(\epsilon)+1$. Hence, on $(\underline{\epsilon}, \bar{\epsilon})$ we have:

$$
\begin{gathered}
\theta^{\prime}(\epsilon) \leq \frac{\beta C(\epsilon) f(\epsilon)}{\lambda^{2} \sigma^{2} \exp \left(-\lambda(1+r+\theta(\epsilon) \epsilon)+\frac{1}{2} \lambda^{2} \sigma^{2} \theta(\epsilon)^{2}\right)} \\
\Rightarrow \theta^{\prime}(\epsilon) \leq \frac{\beta C(\epsilon) f(\epsilon)}{\lambda^{2} \sigma^{2}} \exp \left(\lambda(1+r)+\lambda \theta(\epsilon) \epsilon-\frac{1}{2} \lambda^{2} \sigma^{2} \theta(\epsilon)^{2}\right)
\end{gathered}
$$

But $\lambda \theta(\epsilon) \epsilon-\frac{1}{2} \lambda^{2} \sigma^{2} \theta(\epsilon)^{2} \leq \frac{\epsilon^{2}}{2 \sigma^{2}}$ so that:

$$
\forall \epsilon \in(\underline{\epsilon}, \bar{\epsilon}), \theta^{\prime}(\epsilon) \leq \frac{\beta C(\epsilon) f(\epsilon)}{\lambda^{2} \sigma^{2}} \exp \left(\lambda(1+r)+\frac{\epsilon^{2}}{2 \sigma^{2}}\right)
$$

Hence,

$$
\forall \epsilon \in(\underline{\epsilon}, \bar{\epsilon}), \theta(\epsilon) \leq \theta(\underline{\epsilon})+\int_{\underline{\epsilon}}^{\epsilon} \frac{\beta C(\xi) f(\xi)}{\lambda^{2} \sigma^{2}} \exp \left(\lambda(1+r)+\frac{\xi^{2}}{2 \sigma^{2}}\right) d \xi
$$


This implies that we cannot have $\lim _{\epsilon \rightarrow \bar{\epsilon}} \theta(\epsilon)=+\infty$.

Now, let's consider the remaining possibility that is $\lim _{\epsilon \rightarrow \bar{\epsilon}} \theta(\epsilon)=\theta_{0}(\bar{\epsilon})$. The intuitive reason why this case is also impossible is that the slope when $\theta$ crosses the line associated to the solution $\theta_{0}$ should be infinite and this cannot happen. To see that more precisely let's consider the following ODE:

$$
\epsilon^{\prime}(\theta)=\frac{\lambda^{2} \sigma^{2} \exp \left(-\lambda(1+r+\theta \epsilon(\theta))+\frac{1}{2} \lambda^{2} \sigma^{2} \theta^{2}\right)}{\beta C(\epsilon(\theta)) f(\epsilon(\theta))}\left(\theta-\theta_{0}(\epsilon(\theta))\right)
$$

Let's apply the Cauchy-Lipschitz theorem to the above equation on the domain $\left(\mathbb{R}^{+*}\right)^{2}$ with the Cauchy condition $\epsilon\left(\theta_{0}(\bar{\epsilon})\right)=\bar{\epsilon}$. We have a local solution defined on a small interval $\left[\theta_{0}(\bar{\epsilon})-\eta, \theta_{0}(\bar{\epsilon})+\eta\right]$ and this solution exhibits a local minimum at $\theta_{0}(\bar{\epsilon})$. However, we can build another solution of the above Cauchy problem since the inverse of the maximal solution $\theta$ satisfies the equation and can be prolonged to satisfy the Cauchy condition. Therefore, because of the local minimum, the two solutions are different and this is absurd.

The conclusion is that the maximal interval has no upper bound.

Now, by symmetry the solution is defined on $\mathbb{R}$.

One thing remains to be done. In fact, if we have found a function $\theta(\epsilon)$ that verifies the differential equation and hence a distribution $m$ coherent with the first order condition, we still need to check that the second order condition is verified to be sure that we characterized a maximum of the optimization criterion. This is the purpose of the following proposition:

Proposition 6.4 (Second order condition). Let's introduce

$$
\Gamma(\epsilon, \theta)=-\lambda^{2} \sigma^{2}\left(\theta-\frac{\epsilon}{\lambda \sigma^{2}}\right) \exp \left(-\lambda(1+r)-\lambda \theta \epsilon+\frac{1}{2} \lambda^{2} \theta^{2} \sigma^{2}\right)+\beta m(\theta) C(\epsilon)
$$

Let's consider the unique function $\theta(\epsilon)$, given by the preceding proposition, that satisfies $\forall \epsilon, \Gamma(\epsilon, \theta(\epsilon))=0$ and the conditions of the above proposition.

We have:

$$
\partial_{\theta} \Gamma(\epsilon, \theta(\epsilon))<0
$$

\section{Proof:}

First, let's differentiate the first order condition $\Gamma(\epsilon, \theta(\epsilon))=0$ with respect to $\epsilon$. We get:

$$
\partial_{\epsilon} \Gamma(\epsilon, \theta(\epsilon))+\theta^{\prime}(\epsilon) \partial_{\theta} \Gamma(\epsilon, \theta(\epsilon))=0
$$

Thus, the sign of $\partial_{\theta} \Gamma(\epsilon, \theta(\epsilon))$ is the sign of $-\partial_{\epsilon} \Gamma(\epsilon, \theta(\epsilon))$ and we need to prove that $\partial_{\epsilon} \Gamma(\epsilon, \theta(\epsilon))>0$. 
But:

$\partial_{\epsilon} \Gamma(\epsilon, \theta)=\lambda \exp \left(-\lambda(1+r)-\lambda \theta \epsilon+\frac{1}{2} \lambda^{2} \theta^{2} \sigma^{2}\right)\left(1+\lambda^{2} \sigma^{2} \theta\left(\theta-\frac{\epsilon}{\lambda \sigma^{2}}\right)\right)+\beta m(\theta) C^{\prime}(\epsilon)$

This expression is positive for $\theta=\theta(\epsilon)$ since $\theta(\epsilon) \geq \frac{\epsilon}{\lambda \sigma^{2}}$

\subsection{Example}

Now that existence and uniqueness have been proved, we can try to compute numerically a solution. To know the shape of the curve, it's indeed important to compute the function $\theta(\epsilon)$ for an example and to compare it to the linear function $\theta_{0}(\epsilon)$ we usually obtain in the non-competitive case. This is what we are doing now.

Let us consider the following case. We put $r=2 \%, \sigma=20 \%$ and $\lambda=1$. We put $s=1 \%$ the standard deviation associated to $f$ and we take $\beta$ small. Numerically, we obtain the following result:

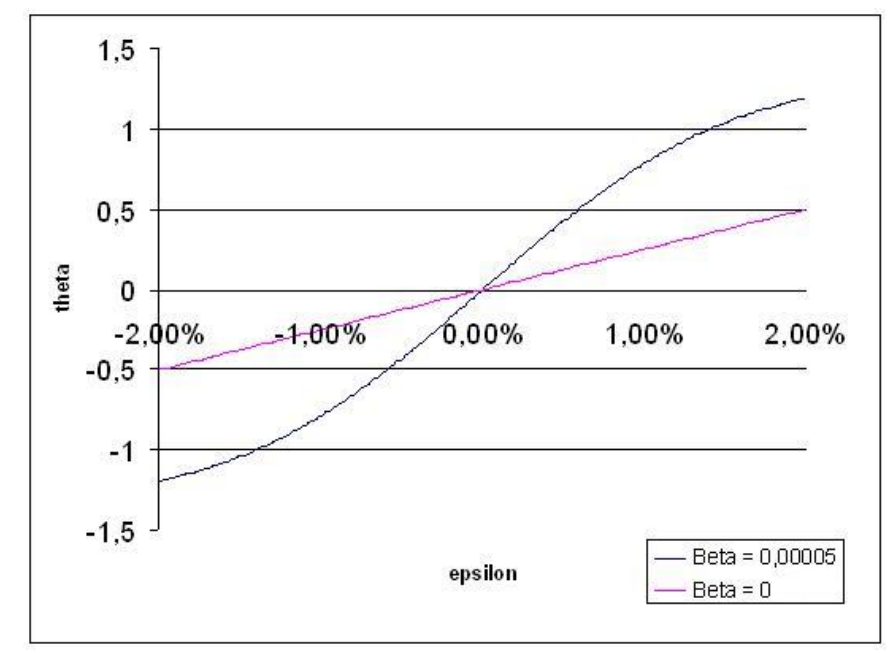

The conclusion is that the introduction of the mean field $m$ overturns the Markowitz model. The Markowitz model indeed supposes that each agent reasons as if he were alone and that's not true in practice. Surprisingly perhaps, even a small influence of competition $(\beta)$ completely changes the shape of the solution and induces asset managers to take risker positions, both bullish and bearish. 


\section{Mean field games model of growth and Pareto distribution of salaries}

\subsection{Introduction to the model mechanisms}

We shall construct an economic growth model based on human capital using the mean field games approach. The idea is to consider a large number of people who will endeavor to increase their human capital in order to increase their salary. Increasing one's human capital usually has two effects: it leads to increased competence and therefore salary, and also, ceteris paribus, a reduction in the number of people one is in competition with and, as a result, an increased salary. To take advantage of these two effects, there is obviously a cost. However, this cost is not the same for each individual since it is a priori easier for someone with poor qualifications to resort to training than for an agent whose human capital is close to what economists call the technology frontier (see [2]).

We consider a large number of agents, each having human capital that we term $q$. This human capital is distributed in the population according to a distribution function we term $m$ (the associated cumulative distribution function is $F$ and $\bar{F}=1-F$ is the tail function).

Let us now define the salary function. If we take a Cobb-Douglas production function (see [22]), it is clear ${ }^{14}$ that the salary can be written in the form:

$$
w(q, m(t, q))= \begin{cases}C \frac{q^{\alpha}}{m(t, q)^{\beta}}, & \text { if } q \text { is in the support of } m(t, \cdot) \\ 0 & \text { otherwise }\end{cases}
$$

If we suppose that $m$ is a distribution function that decreases with $q$, we find the two effects mentioned above.

The costs of increasing human capital must also be made explicit, and we express them as follows:

$$
H\left(\frac{d q}{d t}, \bar{F}(t, q)\right)=\frac{E}{\varphi} \frac{\left(\frac{d q}{d t}\right)^{\varphi}}{\bar{F}(t, q)^{\delta}}, \quad \forall q \text { in the support of } m(t, \cdot)
$$

Here, $E$ is a constant that indicates the inefficiency of the human capital production mechanism and $\delta$ and $\varphi$ are two constants. This functional form means that the cost depends on the growth intensity $a$ of human capital $\left(d q_{t}=a\left(t, q_{t}\right) d t\right)$ but also on the proximity to the technological frontier, because of the tail function $\bar{F}$.

The parameters $\alpha, \beta, \delta$ and $\varphi$ are positive and, to successfully do our calculations, we shall suppose that $\alpha+\beta=\varphi, \beta=\delta$ and $\varphi>1$, thus leaving two

\footnotetext{
${ }^{14}$ In general, if we consider two production factors $x_{1}$ and $x_{2}$, taking a Cobb-Douglas production function means that the production $y$ is of the form $y=A x_{1}^{\alpha_{1}} x_{2}^{\alpha_{2}}$. Hence if $x_{1}$ is labor, the wage simply is $\frac{d y}{d x_{1}}$.
} 
degrees of freedom.

\subsection{The optimization problem and the associated PDEs}

Let us now move on to the problem of optimization of agents. We assume that they will maximize their wealth over time, which is coherent if one is situated in a stylized world without liquidity constraint.

The agents' problem is the following maximization:

$$
\operatorname{Max}_{\left(q_{s}\right), q_{0}=q} \int_{0}^{\infty}\left[w\left(q_{s}, m\left(s, q_{s}\right)\right)-H\left(a\left(s, q_{s}\right), \bar{F}\left(s, q_{s}\right)\right)\right] e^{-r s} d s
$$

To solve this problem, we must first specify the initial distribution of human capital. If we take a Pareto distribution for human capital, i.e. a distribution of the form:

$$
m(0, q)=k \frac{1}{q^{k+1}} 1_{q \geq 1}
$$

we see that the initial distribution of salaries is also a Pareto distribution, which conforms to reality, at least for distribution tails. We therefore opt for this specification.

The optimization problem can be solved, since it is deterministic, by using the classic Euler-Lagrange tools. However, as we shall later introduce uncertainty, we prefer to solve it with mean field games tools.

To do this, we introduce the Bellman function $J$ :

$$
J(t, q)=\operatorname{Max}_{\left(q_{s}\right), q_{t}=q} \int_{t}^{\infty}\left[w\left(q_{s}, m\left(s, q_{s}\right)\right)-H\left(a\left(s, q_{s}\right), \bar{F}\left(s, q_{s}\right)\right)\right] e^{-r(s-t)} d s
$$

The mean field games PDEs that concern $J$ and $m$ are then written in the following form:

$$
w(q, m(t, q))+\partial_{t} J+\operatorname{Max}_{a}\left(a \partial_{q} J-H(a, \bar{F}(t, q))\right)-r J=0
$$

(Kolmogorov) $\quad \partial_{t} m(t, q)+\partial_{q}(a(t, q) m(t, q))=0$

where $a(t, q)=\operatorname{ArgMax}_{a}\left(a \partial_{q} J-H(a, \bar{F}(t, q))\right)$ is the optimal control.

By using the specific forms chosen, we obtain:

$$
\begin{gathered}
C \frac{q^{\alpha}}{m(t, q)^{\beta}}+\frac{\varphi-1}{\varphi} \frac{1}{E^{\frac{1}{\varphi-1}}} \bar{F}(t, q)^{\frac{\beta}{\varphi-1}}\left(\partial_{q} J\right)^{\frac{\varphi}{\varphi-1}}+\partial_{t} J-r J=0 \\
\partial_{t} m(t, q)+\partial_{q}\left(\left(\frac{\bar{F}(t, q)^{\beta}}{E} \partial_{q} J(t, q)\right)^{\frac{1}{\varphi-1}} m(t, q)\right)=0
\end{gathered}
$$


and the optimal control is:

$$
a(t, q)=\left(\frac{\bar{F}(t, q)^{\beta}}{E} \partial_{q} J(t, q)\right)^{\frac{1}{\varphi-1}}
$$

\subsection{Solution}

We can give explicit solutions ${ }^{1516}$ :

Proposition 7.1 (Resolution of the PDEs). If $\varphi(\varphi-1)<\beta k$, there is a unique triple $(J, m, \gamma)$ that satisfies both the PDEs and the additional equation on the optimal control function: $a(t, q)=\gamma q$.

Solutions are of the following form:

$$
\begin{gathered}
m(t, q)=k \frac{\exp (\gamma k t)}{q^{k+1}} 1_{q \geq \exp (\gamma t)} \\
J(t, q)=B \exp (-\beta k \gamma t) q^{\beta k+\varphi} 1_{q \geq \exp (\gamma t)}
\end{gathered}
$$

where $\gamma$ and $B$ are related by $\gamma=\left(\frac{B}{E}(\beta k+\varphi)\right)^{\frac{1}{\varphi-1}}$

\section{Proof:}

First of all, the additional condition is equivalent to a constant growth rate for $q_{t}$ and therefore, we obtain the Pareto distribution $m(t, \cdot)$ stated above.

Therefore, we have the following equation for $\partial_{q} J(t, q)$ if $q \geq \exp (\gamma t)$ :

$$
\partial_{q} J(t, q)=E(\gamma q)^{\varphi-1} \bar{F}(t, q)^{-\beta}=E(\gamma q)^{\varphi-1} e^{-\beta k \gamma t} q^{\beta k}
$$

Hence (the constant being zero),

$$
J(t, q)=\frac{E}{\beta k+\varphi} \gamma^{\varphi-1} e^{-\beta k \gamma t} q^{\beta k+\varphi}
$$

If we plug this expression into the Hamilton Jacobi Bellman equation we get:

$$
\begin{gathered}
\frac{C}{k^{\beta}} q^{\beta k+\varphi} e^{-\beta k \gamma t}+\frac{\varphi-1}{\varphi} E \gamma^{\varphi} q^{\beta k+\varphi} e^{-\beta k \gamma t} \\
-\beta k \gamma \frac{E}{\beta k+\varphi} \gamma^{\varphi-1} e^{-\beta k \gamma t} q^{\beta k+\varphi}-r \frac{E}{\beta k+\varphi} \gamma^{\varphi-1} e^{-\beta k \gamma t} q^{\beta k+\varphi}=0
\end{gathered}
$$

From this we get:

$$
\frac{C}{k^{\beta}}+\frac{\varphi-1}{\varphi} E \gamma^{\varphi}-\beta k \frac{E}{\beta k+\varphi} \gamma^{\varphi}-r \frac{E}{\beta k+\varphi} \gamma^{\varphi-1}=0
$$

\footnotetext{
${ }^{15}$ There are some additional restrictions about the parameters for the integral in the criterion to be defined at equilibrium (see [22])

${ }^{16}$ As always, this solution of the PDEs does not automatically induce a solution of the control problem and a verification theorem still need to be written.
} 


$$
\frac{C}{k^{\beta}}+\frac{(\varphi-1) \varphi-k \beta}{\phi(k \beta+\phi)} E \gamma^{\varphi}-r \frac{E}{\beta k+\varphi} \gamma^{\varphi-1}=0
$$

Since $\varphi(\varphi-1)<\beta k, \gamma$ is unique.

Even though we cannot prove that there is uniqueness, this solution is very interesting since $\gamma$ corresponds in fact to the rate of growth of human capital, which is the same for everyone. Furthermore, we see that the solution $m$ is always Pareto-type, which is remarkable since in practice salary distribution tails are indeed Pareto-type (see [7, 8, 48, 49] for more details on the analysis of wealth distribution).

\subsection{Underlying mechanisms}

The fact that there is a regular growth path merits spending a few moments on the underlying economic mechanism. To begin with, the basic reason why people change their human capital is due to two effects. First, there is a pure wage effect since, ceteris paribus, wage increases with human capital. However, this effect cannot explain by itself the continuous improvement of human capital at a constant growth rate. The effect needed to ensure a convincing explanation is an escape competition effect ${ }^{17}$. A given individual taken at random in the population is threaten by people who have less human capital than he has (say $\tilde{q})$. Indeed, if part of those people were to improve there human capital so that they end up with a human capital $\tilde{q}$ they would compete with our individual on the labor market, reducing her wage. This effect is the origin of continuous growth in our model. We have here a continuum of agents and therefore, for any given individual, there is always a threat ${ }^{18}$. We think therefore that the Schumpeterian effect which basically assumes that people will not improve their human capital if the gains are too small is reduced to nothing because there is always a potential competitor and that's why a Darwinian effect (competition effect) dominates. Let's indeed highlight how tough is the threat effect. Each agent knows that every one is threaten by every one, and that fear will induce behaviors that will make the frightening event happen and be more important.

This model shows that the growth process is not only due to those who innovate, that is to say "researchers" near the technological frontier, but is in fact a process that involves the whole population and is fostered by those who are far from the technological frontier and threaten the leaders by improving their human capital. The process revealed is therefore very mean field games, if we can put it like that, since it brings into play an overall social dimension.

\footnotetext{
${ }^{17}$ see $[6,5,4]$ for the link between growth and competition

${ }^{18}$ In practice everybody thinks there are people less gifted than he is...
} 


\subsection{A stochastic generalization}

Let us now move on to a more stochastic model.

We suppose that $d q_{t}=a\left(t, q_{t}\right) d t+\sigma q_{t} d W_{t}$ where $W$ s a Brownian common to all the agents. If therefore we put $q^{m}$ as the minimum human capital (this is in fact a new state variable that evolves according to $d q_{t}^{m}=a\left(t, q_{t}^{m}\right) d t+\sigma q_{t}^{m} d W_{t}$ where $a$ is here the optimal control ), we see that the Bellman function can be written $J=J\left(t, q, q^{m}\right)$ and the PDEs are:

$$
\begin{gathered}
\max _{a} C \frac{q^{\alpha}}{m(t, q)^{\beta}}-\frac{E}{\varphi} \frac{a^{\varphi}}{\bar{F}(t, q)^{\beta}}-r J \\
+\partial_{t} J+a \partial_{q} J+\frac{\sigma^{2}}{2} q^{2} \partial_{q q}^{2} J+a^{\prime} \partial_{q^{m}} J+\frac{\sigma^{2}}{2} q^{m 2} \partial_{q^{m} q^{m}}^{2} J+\sigma^{2} q q^{m} \partial_{q q^{m}}^{2} J=0
\end{gathered}
$$

where $a^{\prime}$ is nothing but $a\left(t, q_{t}^{m}\right)$, exogenous in the optimization.

The optimal control is given by:

$$
a(t, q)=\left(\frac{\bar{F}(t, q)^{\beta}}{E} \partial_{q} J(t, q)\right)^{\frac{1}{\varphi-1}}
$$

Lemma 7.2. If $a(t, q)=\gamma q$, then the probability distribution function of the $q$ 's is $m(t, q)=k \frac{\left(q_{t}^{m}\right)^{k}}{q^{k+1}} 1_{q \geq q_{t}^{m}}$.

\section{Proof:}

Assuming $a(t, q)=\gamma q$ we get:

$$
\begin{gathered}
q_{t}=q_{0} \exp \left(\left(\gamma-\frac{\sigma^{2}}{2}\right) t+\sigma W_{t}\right)=q_{0} q_{t}^{m} \\
\Rightarrow m(t, q)=k \frac{\exp \left(k\left(\gamma-\frac{\sigma^{2}}{2}\right) t+\sigma k W_{t}\right)}{q^{k+1}} 1_{q \geq \exp \left(\left(\gamma-\frac{\sigma^{2}}{2}\right) t+\sigma W_{t}\right)}=k \frac{\left(q_{t}^{m}\right)^{k}}{q^{k+1}} 1_{q \geq q_{t}^{m}}
\end{gathered}
$$

Proposition 7.3 (Resolution of the $\mathrm{PDEs}^{19}$ ). If $\varphi(\varphi-1)<\beta k$ and $r>$ $\frac{\sigma^{2}}{2} \varphi(\varphi-1)$, then, there is a unique growth rate $\gamma$ compatible with the problem and $J$ is of the form:

$$
J\left(q, q^{m}\right)=B q^{\beta k+\varphi}\left(q^{m}\right)^{-\beta k} 1_{q \geq q^{m}}
$$

where $\gamma$ and $B$ are related by $\gamma=\left(\frac{B}{E}(\beta k+\varphi)\right)^{\frac{1}{\varphi-1}}$

Moreover, $\gamma$ is given by:

$$
\frac{\varphi(\varphi-1)-\beta k}{\varphi} \gamma^{\varphi}=\left(r-\varphi(\varphi-1) \frac{\sigma^{2}}{2}\right) \gamma^{\varphi-1}-\frac{C(\varphi+\beta k)}{E k^{\beta}}
$$

${ }^{19}$ For the "transversality" condition, see [22] 


\section{Proof:}

First, if $a(t, q)=\gamma q$ then,

$$
\partial_{q} J\left(t, q, q^{m}\right)=E(\gamma q)^{\varphi-1} \bar{F}(t, q)^{-\beta}=E \gamma^{\varphi-1} q^{\beta k+\varphi-1}\left(q_{t}^{m}\right)^{-\beta k}
$$

From this we deduce that the solution is of the stated form with $B=\frac{E}{\beta k+\varphi} \gamma^{\varphi-1}$.

If we want to find $B$ or $\gamma$ we need to plug the expression for $J$ in the Hamilton Jacobi Bellman equation. This gives:

$$
\begin{gathered}
q^{\beta k+\varphi-1}\left(q^{m}\right)^{-\beta k}\left[\frac{C}{k^{\beta}}-\frac{E}{\varphi} \gamma^{\varphi}-r B+\gamma(\beta k+\varphi) B-\beta k \gamma B\right. \\
\left.+\frac{\sigma^{2}}{2} B((\beta k+\varphi)(\beta k+\varphi-1)+(-\beta k)(-\beta k-1)+2(\beta k+\varphi)(-\beta k))\right]=0 \\
\frac{C}{k^{\beta}}-\frac{E}{\varphi} \gamma^{\varphi}+\gamma \varphi B-\left(r-\varphi(\varphi-1) \frac{\sigma^{2}}{2}\right) B=0 \\
\frac{C(\beta k+\varphi)}{E k^{\beta}}-\frac{\beta k+\varphi}{\varphi} \gamma^{\varphi}+\varphi \gamma^{\varphi}-\left(r-\varphi(\varphi-1) \frac{\sigma^{2}}{2}\right) \gamma^{\varphi-1}=0 \\
\frac{\varphi(\varphi-1)-\beta k}{\varphi} \gamma^{\varphi}=\left(r-\varphi(\varphi-1) \frac{\sigma^{2}}{2}\right) \gamma^{\varphi-1}-\frac{C(\varphi+\beta k)}{E k^{\beta}}
\end{gathered}
$$

As before, it is clear that, given our hypotheses, this equation has a unique solution.

One consequence of these solutions is that growth is greater in the presence of a risk factor, even though this risk is common to everyone. This confirms the fact that growth is fostered by the fear to be overcome.

\section{Mathematical perspectives}

The examples above clearly show that many kinds of nonlinear problems arise in the context of mean field games models. For most of them, these nonlinear problems are new systems of coupled nonlinear equations which, in the case the state of the agents is described by continuous variables and the time variable is continuous, are partial differential equations. In all situations, the main novelty of these systems is the mixed "forward-backward" nature of the equations composing these systems. In general, no classical mathematical theory could be involved to tackle them. Furthermore, in the "partial differential" case for instance, the scope of the necessary new mathematical theory is quite large since many classical Partial Differential Equations (such as Hamilton Jacobi Bellman equations, Nonlinear heat or porous media equations, kinetic equations such as Vlasov or Boltzmann equations, compressible Euler equations of Fluid Mechanics, general semilinear elliptic equations, Hartree equations in Quantum 
Mechanics, optimal transportation problems, ... ) are in fact particular case of mean field games systems! This is to be expected since all these models arise in Mechanics and Physics model in a "mean field" fashion where the mean field sum up the collective or average behaviour of a large number of interacting particles (which can be seen as agents without any possibility of choosing their actions !)

Both the novelty of the mean field games models and the "range" of problems explain why numerous (and delicate) mathematical issues are being raised by mean field games theory.

To conclude, we set a brief (and thus far from exhaustive) list of issues for which some mathematical understanding is available (although a lot of open questions remain):

\section{- Justification of the derivation of mean field games models from N-player Nash equilibria:}

A general analytical framework has been developed by J.-M. Lasry and P.-L. Lions ([34, 35, 36, 37] and [38]) that allows to derive rigorously the mean field games equations from N-player Nash equilibria. This framework is of independent mathematical interest and has many other applications (limits of equations when the dimension goes to infinity, interacting particle systems, large deviations for stochastic partial differential equations, ... )

\section{- Expansion in $\mathrm{N}$ as the number of players $\mathrm{N}$ goes to infinity:}

Such an expansion has been rigorously established for a large class of examples of mean field games models (at least in situations where the uniqueness of solutions holds for the limit mean field games system).

- Existence and regularity results:

For large classes of models, the existence and regularity of solutions is now understood.

- Uniqueness results:

Two uniqueness regimes have been identified: the case of a small horizon and the case of a "monotone" coupling. In addition, non-uniqueness examples are available that show that there does not seem to be any other general uniqueness regime.

\section{- Stability questions:}

Of course, closely related to uniqueness is the issue of the stability of solutions which is indeed, true in the uniqueness regimes. It is worth pointing out that there are many notions of stability (small perturbations of data, horizon going to infinity, ...) which are all of interest.

- Interpretation of mean field games models as control problems:

For a substantial class of mean field games models, it is possible to show that 
the mean field games system corresponds to a global optimal control problem of a certain partial differential equation. Roughly speaking, the system is then described as the coupling of the equations governing the state of a system and its dual state.

\section{- Numerical Approaches:}

Various numerical methods or approaches have been proposed such as direct discretizations (finite elements) of the systems, discretization of the associated control problem (when there is one, see above), various iteration strategies, or the addition of an extra time variable ("relaxation time").

\section{- Limiting situations:}

One relevant class of limiting situations corresponds to what could be called a planning problem. Instead of prescribing the initial state ("density") of the agents population and the terminal "cost-reward" profile for each agent as it is the case for "classical" mean field games models, one prescribes the state of the population (agents) both initially and at the end of the time interval (in other words, at both ends). In that case, the unknown terminal "cost-reward" function can be thought as the incentive scheme for each player which will lead to the desired final state of the population. Most of the preceding mathematical results can now be extended to that "limiting" class of models. 


\section{References}

[1] M. Abramowitz and I. Stegun. Handbook of Mathematical Functions with Formulas, Graphs, and Mathematical Tables. Dover, 1964.

[2] D. Acemoglu, P. Aghion, and F. Zilibotti. Distance to frontier, selection, and economic growth. Journal of the European Economic Association, 4(1), Mar. 2006.

[3] Y. Achdou and I. Capuzzo-Dolcetta. Mean field games: Numerical methods. To appear.

[4] P. Aghion, N. Bloom, R. Blundell, R. Griffith, and P. Howitt. Competition and innovation: An inverted-u relationship. Quarterly Journal of Economics, 120(2), May. 2005.

[5] P. Aghion, C. Harris, P. Howitt, and J. Vickers. Competition, imitation and growth with step-by-step innovation. The Review of Economic Studies, 68(3), Jul. 2001.

[6] P. Aghion and P. Howitt. Forthcoming book.

[7] A. Atkinson. Top incomes in the united kingdom over the 20th century. Journal of the Royal Statistical Society, 168, 2005.

[8] A. Atkinson and T. Piketty. Top Incomes over the Twentieth Century: A Contrast between European and English-Speaking Countries. Oxford University Press, 2007.

[9] R. Aumann. Markets with a continuum of traders. Econometrica, 32(1/2), 1964.

[10] M. Bardi and I. Capuzzo-Dolcetta. Optimal Control and Viscosity Solutions of Hamilton- Jacobi-Bellman equations. Birkhäuser, Boston, 1997.

[11] A. Bensoussan and J. Frehse. Nonlinear elliptic systems in stochastic game theory. J. Reine Angew. Math., 1984.

[12] A. Bensoussan and J. Frehse. Ergodic bellman systems for stochastic games in arbitrary dimension. Proc. Roy. Soc. A., 1995.

[13] G. Carmona. Nash equilibria of games with a continuum of players. Preprint, 2004.

[14] B. K. Chakrabarti, A. Chatterjee, and S. Yarlagadda. Econophysics of Wealth Distributions. Springer-Verlag, 2005.

[15] S. Cordier, L. Pareschi, and G. Toscani. On a kinetic model for a simple market economy. Journal of Statistical Physics, (1/2), 2005.

[16] R. Cross, M. Grinfeld, and H. Lamba. A mean-field model of investor behaviour. Journal of Physics: Conference Series 55, 2006. 
[17] H. A. David and H. N. Nagaraja. Order Statistics (Third edition). Wiley, 2003.

[18] Ivar Ekeland. Le pétrole sera-t-il bradé ? Pour la Science, 2007.

[19] I. Farkas, D. Helbing, and T.Vicsek. Mexican wave in an excitable medium. Nature, 2002.

[20] P.-N. Giraud, O. Guéant, J.-M. Lasry, and P.-L. Lions. A mean field game model of oil production in presence of alternative energy producers. To appear.

[21] D. Gomes, J. Mohr, and R. Sousa. Discrete time, finite state space mean field games.

[22] O. Guéant. Mean Field Games and applications to economics. PhD thesis, Université Paris-Dauphine, 2009.

[23] O. Guéant. A reference case for mean field games. Journal de Mathématiques Pures et Appliquées, 2009.

[24] O. Guéant. A reference case for mean field games. Cahier de la Chaire Finance et Développement Durable, 10, 2009.

[25] Olivier Guéant, Jean-Michel Lasry, and Pierre-Louis Lions. Mean field games and oil production. Finance and Sustainable Development : Seminar's lectures., To appear in 2009.

[26] Roger Guesnerie. An exploration of the eductive justifications of the rational-expectations hypothesis. The American Economic Review, 82(5), Dec. 1992.

[27] U. Horst. Ergodic fluctuations in a stock market model with interacting agents: the mean field case. Discussion paper No. 106, Sonderforschungbereich 373, Humboldt Universität, Berlin, 1999.

[28] H. Hotelling. The economics of exhaustible resources. The Journal of Political Economy, 39(2), 1931.

[29] E. Kalai. Private information in large games. 2000.

[30] E. Kalai. Ex-post stability in large games. 2001.

[31] E. Kalai. Large robust games. 2002.

[32] A. Khan and Y. Sun. Non-cooperative games with many players. 2002.

[33] A. Lachapelle, J. Salomon, and G. Turinici. Computation of mean field equilibria in economics. To be published, 2009.

[34] J.-M. Lasry and P.-L. Lions. Jeux à champ moyen i. le cas stationnaire. C. R. Acad. Sci. Paris, 343(9), 2006. 
[35] J.-M. Lasry and P.-L. Lions. Jeux à champ moyen ii. horizon fini et contrôle optimal. C. R. Acad. Sci. Paris, 343(10), 2006.

[36] J.-M. Lasry and P.-L. Lions. Mean field games. Japanese Journal of Mathematics, 2(1), Mar. 2007.

[37] J.-M. Lasry and P.-L. Lions. Mean field games. Cahiers de la Chaire Finance et Développement Durable, (2), 2007.

[38] P.-L. Lions. Théorie des jeux à champs moyen et applications. Cours au Collège de France, http://www.college-defrance.fr/default/EN/all/equ_der/cours_et_seminaires.htm, 2007-2008.

[39] P.-L. Lions. Mathematical Topics in Fluid Mechanics. Oxford Science Publications, Clarendon Press, Oxford, Vol. 1 (1996) ; Vol. 2 (1998).

[40] R. Lucas and T. Sargent. Rational expectations and econometric practice. Univ of Minnesota Press, 1981.

[41] H. Markowitz. Portfolio selection. The Journal of Finance, 7(1), Mar. 1952.

[42] John F. Muth. Rational expectations and the theory of price movements. Econometrica, (3), 1961.

[43] J. Nash. The bargaining problem. Econometrica, 1950.

[44] J. Nash. Equilibrium points in n-person games. Proceedings of the National Academy of Sciences of the United States of America, 1950.

[45] J. Nash. Non-cooperative games. Annals of Mathematics, 1951.

[46] J. Nash. Two-person cooperative games. Econometrica, 1953.

[47] J. Von Neumann and O. Morgenstern. Theory of Games and Economic Behavior. Princeton University Press, 1944.

[48] T. Piketty. Income inequality in france, 1901-1998. Journal of Political Economy, 111(5), 2003.

[49] T. Piketty and E. Saez. Income inequality in the united states, 1913-1998. Quarterly Journal of Economics, 118(1), Feb. 2003.

[50] N. Touzi. Stochastic control and application to finance. Scuola Normale Superiore, Pisa. Special Research Semester on Financial Mathematics, 2002. 